\title{
A free-boundary model for diffusion-induced grain boundary motion
}

\author{
P. C. FIFE ${ }^{\dagger}$ \\ Mathematics Department, University of Utah, 155 So. 1400 East, Salt Lake City, \\ UT 84112-0090, USA
}

J. W. CAHN

Materials Science and Engineering Laboratory, NIST, Gaithersburg, MD 20899-8555, USA

AND

C. M. Elliott

Centre for Mathematical Analysis and its Applications, University of Sussex, Falmer, Brighton BNI 9QH, UK

[Received 10 March 2000 and in revised form 17 November 2000]

\begin{abstract}
On the basis of a phase field model previously proposed in Cahn et al. (Acta Mater. 45, 43974413 (1997)) to describe the phenomenon of diffusion-induced grain boundary motion (DIGM), we present a formal asymptotic reduction to a moving free-boundary problem. This problem is one of enhanced motion by curvature. The enhancement depends on the local concentration jump, across the grain boundary, of a solute species that is diffusing along the boundary. The reduction depends on the material diffusivity vanishing outside the grain boundary; this also introduces important mathematical and conceptual difficulties which are addressed in detail.

A rigorous theory is given for two-dimensional solutions of the free-boundary problem when there is steady motion of the grain boundary spanning a plate from face to face, solute being supplied at the faces of the plate. The motion is in the direction parallel to the face. This situation represents well known experiments designed to illustrate DIGM. The corresponding theory is also given for grain boundaries which do not span the plate, but rather trail behind the moving intersection with one of the faces, never reaching the other one.
\end{abstract}

Keywords: Grain boundary motion; diffusion; free-boundary problem; motion by curvature; asymptotics

\section{Introduction}

A common experimental observation in materials science is that boundaries between crystalline grains (grain boundaries) in a metallic alloy support enhanced diffusion of atoms of the alloy species, and the presence of concentration gradients in these boundaries may induce the migration of the boundary itself. This effect, which has been thoroughly studied, is called diffusion-induced grain boundary motion (DIGM) (see the review [13], the other references in [4], and especially the commentary in Section 1 of [3]).

Various theoretical explanations have been given. In particular, some of the present authors, with Penrose, developed a phase field model in [4] based on a gradient flow with respect to a proposed

†Email: fife@math.utah.edu 
free-energy functional that depends on the concentration distribution $c$ of a solute species in the grain boundary, an order parameter $\phi$ relevant to the local crystallinity, and spatial gradients of the latter. We showed that there was no motion unless free-energy interaction terms between $c$ and $\phi$ were introduced. These are terms in the free energy which depend on both $c$ and $\phi$.

The lowest-order interaction term that was found to support DIGM represented an elastic effect, and it led to a forcing term for the motion. Experiments have shown that the elastic energy due to composition inhomogeneities in the crystals gives rise to the principal driving force for DIGM $[18,19]$.

Our analysis was limited to a simple thin-plate geometry, in which the grain boundary spanning the plate remained planar at all times. This is a reasonable assumption consistent with experiments on thin plates $(\mathrm{O}(10 \mu \mathrm{m}))$. Experiments have shown, however, that for thicker plates the trace of the grain boundary as seen on cross sections perpendicular to the plate's surface is curved.

In this paper (and in [3]) the assumption that the boundary is planar will be avoided. The mathematics will be made tractable by means of a sharp-interface approximation based on the phase field model of DIGM given in [4], with the assumption that the driving force is the elastic interaction. The detailed derivation of this approximation is given in Section 2.

The model we obtain is a free-boundary problem in the plane for a mobile curve or interface representing the moving grain boundary. It takes the form of a forced motion by curvature problem, the force depending on the local concentration of a solute species in the grain boundary. In addition, the solute diffuses along the moving interface from a source (or sink) on the faces of the plate and is absorbed into the growing grain (or depleted from the shrinking grain) by the motion of the grain boundary.

The model is derived by means of formal asymptotics involving a small parameter $\epsilon$ representing the strength of the elastic interaction responsible for DIGM, and another (possibly small) parameter $\rho$, the latter being a combination of $\epsilon$ and various material constants. There are formidable mathematical and conceptual complications having to do with the vanishing of the material diffusivity outside the grain boundary. These difficulties are addressed in detail in Section 2.3.2 and Appendix B. Theorems 3 and 4 in that appendix provide a conceptual basis for the definition and properties of solutions of a wide class of differential equations with degenerate diffusion, extending beyond the present context.

Three versions of the free-boundary problem are derived in Sections 2.4, 2.6, and 2.7. The second version is a higher-order (in $\epsilon$ ) version of the first. In simple cases the third version constitutes an equation for the quick redistribution of an arbitrary initial solute distribution to become compatible with the version in Section 2.4.

There is a special class of steady problems which is of great interest. These problems have to do with experimental setups involving multigrain metallic plates with two kinds of surfaces: the surfaces of the plates are adjacent to a vapour which acts as source or sink of solute atoms. The grain boundaries, the surfaces between grains, are mobile and provide paths for the diffusion to or from the vapour. Although there are junctions among grain boundaries of several grains and where grain boundaries intersect the plate faces, we consider only two grains and a single grain boundary that spans the plate.

When the motion is steady, that class of problems can be formulated as a system of ordinary differential equations for the determination of the distribution of solute and shape of the boundary. The imposed boundary conditions would make it overdetermined, were it not for the presence of an unknown parameter (velocity) which must also be determined. There are only two (dimensionless) fixed parameters in these equations: the thickness of the plate and the parameter $\rho$ mentioned before. 
These steady problems are investigated rigorously in Section 3. Conditions for the existence of solutions are given, and the case when $\rho \ll 1$ is explored.

We will assume such a steady travelling wave in a thin specimen which is infinite in extent in which the experiment has been conducted for an infinite time. Obviously this is an idealization; in the laboratory all specimens are finite and experiments last a finite amount of time. In order to have a travelling wave DIGM problem, it is necessary to assume that the diffusion constant of the crystalline grains filling the space between the surfaces have a vanishingly small diffusion coefficient. Otherwise ordinary diffusion would have filled the grains in the infinite amount of time before the present. In reality there is diffusion in the grains, but it is much smaller than in the grain boundaries, and can be shown to have little effect in the time of a typical DIGM experiment.

A discussion of the results of the paper, including a review of order of magnitude assumptions, is given in Section 4.

We note that travelling wave solutions for forced mean curvature flow with prescribed forcing have previously been studied in various settings (see $[5,6])$. Numerical calculations based on both phase-field and sharp-interface models support the formal asymptotics of this paper and yield for both models travelling wave solutions in appropriate parameter regimes as the long-time limit of initial value problems $[7,15]$.

In [3] further results on the model derived here are given. In addition, the effects of various boundary conditions imposed by the physics at the junctions of the grain boundary with the plate surfaces are examined. One of these, which considers grooving at this moving junction by surface diffusion, has a profound effect on the solutions.

Recently an existence/uniqueness theory for a version of the phase field model of DIGM was given in [8].

\section{The sharp-interface model}

\subsection{Formulation and scaling}

The phase field model described in [4] uses two field variables: $\hat{c}$, representing the concentration of solute atoms in the metal plate, and an order parameter $\phi$ which takes the value +1 in one of the crystal grains, -1 in the other, and intermediate values in the grain boundary. We use the symbol $\hat{x}$ for (two-dimensional) space and $\hat{t}$ for dimensional time. The field variables obey the kinetic equations

$$
\begin{aligned}
& \tau \frac{\partial \phi}{\partial \hat{t}}= \phi+\delta^{2} \hat{\nabla}^{2} \phi-\hat{\epsilon} \frac{\partial \hat{p}(\phi, \hat{c})}{\partial \phi}, \quad \text { if }-1<\phi<1 ; \quad \text { else } \frac{\partial \phi}{\partial \hat{t}}=0, \\
& \quad \phi \text { continuously differentiable in }(\hat{x}, \hat{t}) \\
& \frac{\partial \hat{c}}{\partial \hat{t}}=\hat{\nabla} \cdot[\hat{D}(\phi) \hat{\nabla} \hat{w}], \\
& \hat{w}=\hat{c}+\hat{\epsilon} \beta \frac{\partial \hat{p}(\phi, \hat{c})}{\partial \hat{c}},
\end{aligned}
$$

where $\hat{\nabla}$ is with respect to $\hat{x}, \tau$ is a constant which can be thought of as a relaxation time for the grain boundary motion, $\delta$ is a constant length associated with gradient contributions to the free-energy density of the system, $\hat{D}(\phi)$ is the diffusivity, $\hat{\epsilon}$ is a scaled dimensionless stress that results from a unit composition change, and $\beta$ is a material parameter, defined in (2.4), representing an interfacial 
component of the chemical potential. Roughly, it is the ratio of interfacial energy per surface atom to chemical energy, also per atom. It will turn out that $\delta$ is comparable with the thickness of the grain boundary.

Equations (2.1)-(2.4) arise from a double-obstacle variational inequality framework, in which $\phi$ is restricted by $-1 \leqslant \phi \leqslant 1$. In [4], they were derived as a relaxation (gradient) flow for a freeenergy integral containing an interaction term $\hat{\epsilon} \hat{p}(\phi, \hat{c})$. The elastic interaction will be modelled as in [4] by taking

$$
\hat{p}(\phi, \hat{c})=\frac{1}{8}(1+\phi)^{2}\left(\hat{c}-\hat{c}_{0}\right)^{2}
$$

The interaction term $\hat{p}$ given in (2.5) differs slightly in appearance $((1+\phi)$ versus $(1-\phi))$ from that in [4], because in that paper the leading edge of the grain boundary was taken to be where $\phi=-1$. In this paper we use a different convention, characterizing the leading edge by $\phi=1$ and the trailing edge by $\phi=-1$.

No travelling wave solution (not even a standing wave solution) exists if $\hat{\epsilon}=0$, from which we concluded in [4] that the interaction term $\hat{\epsilon} \hat{p}$ must be nonzero to produce steady grain boundary movement.

The diffusion coefficient $\hat{D}$ will be zero outside the grain boundary, i.e. where $\phi= \pm 1$, and positive inside; in fact for some $v \geqslant 0, b>0$, we assume that $\hat{D}(\phi)$ is a monotone increasing (decreasing) function of $\phi$ for $\phi$ near $-1(+1)$, that

$$
\hat{D}(\phi)=b\left(\phi^{2}-1\right)^{v}(1+o(1)), \quad \phi \rightarrow \pm 1
$$

and that this relation may be differentiated with respect to $\phi$. The function $\hat{D}(\phi)$ is allowed to be discontinuous at the edges $\phi= \pm 1$ (this is the case $v=0$ in (2.6)).

The degeneracy of $\hat{D}$ where $\phi= \pm 1$ means that the concept of solution of (2.3) is ambiguous; this is associated with the fact that solutions generally have singularities where the degeneracy takes place. To remove this ambiguity, we must supplement that equation with extra requirements. Since (2.3) expresses conservation of solute, we require first of all that $\hat{c}$ be conserved at the possible singularities, namely at the edges of the grain boundary. Secondly, we shall require that our solutions be limits of solutions of nonsingular problems associated with diffusivities $\hat{D}_{n}$ which are strictly positive and continuous, and which approach $\hat{D}$ as $n \rightarrow \infty$. Among other things, these limiting solutions are such that $\hat{w}, \hat{c}$, and $\hat{D} \nabla \hat{w}$ are continuous at the trailing edge of the grain boundary, where $\phi=-1$. Sections 2.3.2, 2.4, and Appendix B are in large part devoted to these issues. See also the last part of Section 2.2.

The conditions in the undisturbed part of the metal, just in front of the moving grain boundary, are taken to be $\hat{c}=\hat{c}_{0}, \phi=1$. Just behind it, we have $\phi=-1$ and $\hat{c}$ is to be determined. In this paper we shall assume that $\hat{c}_{0}=0$. If this is not the case, then easy adjustments to the analysis can be made.

We now nondimensionalize equations (2.1)-(2.4).

Let $c^{\dagger} \leqslant 1$ be the maximal value of $\hat{c}$ attained in the process and $D_{m} / \pi=\bar{D}$ be a certain average value of the function $\hat{D}(\phi)$, to be given later (2.71). Normalized quantities $c, w, \epsilon, D, p$ are obtained by setting

$$
\hat{c}=c^{\dagger} c, \quad \hat{w}=c^{\dagger} w, \quad \epsilon=\frac{\hat{\epsilon}\left(c^{\dagger}\right)^{2}}{\pi}, \quad \hat{D}(\phi)=D_{m} D(\phi), \quad \hat{p}=\frac{\left(c^{\dagger}\right)^{2}}{\pi} p .
$$


We use the dimensionless combinations

$$
A=\frac{\delta^{2}}{D_{m} \tau}, \quad \rho=\frac{\epsilon}{A},
$$

and set

$$
\hat{x}=\frac{\delta}{\epsilon} x, \quad \hat{t}=\frac{\tau}{\rho \epsilon^{2}} t .
$$

Making these substitutions, we arrive at the equations

$$
\begin{gathered}
\rho \epsilon^{2} \phi_{t}=\phi+\epsilon^{2} \nabla^{2} \phi-\epsilon p_{\phi}, \quad-1<\phi<1, \\
\nabla \cdot D \nabla w=\epsilon c_{t}, \\
w=c+\frac{\hat{\epsilon} \beta}{\pi} p_{c},
\end{gathered}
$$

and (2.2). The needed supplementary conditions will be considered in Section 2.2.

Our asymptotics will be based on the following assumptions about the parameters:

$$
\hat{\epsilon} \beta \ll 1, \quad c^{\dagger} \sqrt{\hat{\epsilon} A} \ll 1, \quad \frac{\hat{\epsilon}\left(c^{\dagger}\right)^{2}}{A} \leqslant \mathrm{O}(1),
$$

which are equivalent to the relations

$$
\hat{\epsilon} \beta \ll 1, \quad \rho \leqslant \mathrm{O}(1), \quad \epsilon \rho^{-1 / 2} \ll 1 .
$$

These relations are in accord with typical values of the parameters (see Section 4). Here and throughout the paper, relations such as ' $\leqslant \mathrm{O}(1), \leqslant \mathrm{O}(\epsilon)$ ', etc. will mean that there exists a constant $C$ of moderate size, whose value will change from context to context, independent of $\epsilon$, such that the quantity on the left of the inequality is $\leqslant C, \leqslant C \epsilon$, etc. in absolute value. Similar meanings will be given to notation such as $\leqslant \mathrm{O}(\eta)$, where $\eta$ is any other parameter. We shall use such generic constants $C$ in other contexts as well.

In addition to (2.14), which concern the material parameters, the analysis will operate under certain assumptions, given in Section 2.3, on the solutions we are constructing, namely about the orders of magnitude of the derivatives appearing in (2.10), (2.11). These assumptions enable the formal analysis to proceed in a logical manner, so that our formal construction provides solutions that have the assumed properties.

We restrict attention to two-dimensional problems, the grain boundary at time $t$ being represented by a mobile curved strip $G(t)$ in the $x=\left(x_{1}, x_{2}\right)$ plane bounded on two sides by curves $\Gamma_{ \pm}(t)$. (The picture in three-space will have the grain boundary extending with no change infinitely far in the positive and negative $x_{3}$ directions.) The two grains lie on either side of this strip; we represent them by the two domains $\Omega_{+}$(where $\phi=1$ ) and $\Omega_{-}$(where $\phi=-1$ ). The curves $\Gamma_{ \pm}$ are the common boundaries between $G$ and $\Omega_{ \pm}$. See Fig. 1 .

By definition, $G$ is linked to the phase function by the relations

$$
G(t)=\{x:-1<\phi(x, t)<1\},
$$

and on the boundary $\Gamma_{+}(t) \cup \Gamma_{-}(t)$ of $G(t)$,

$$
\phi(x, t)= \pm 1, \quad \nabla \phi(x, t)=0 \quad \text { for } \quad x \in \Gamma_{ \pm}(t),
$$




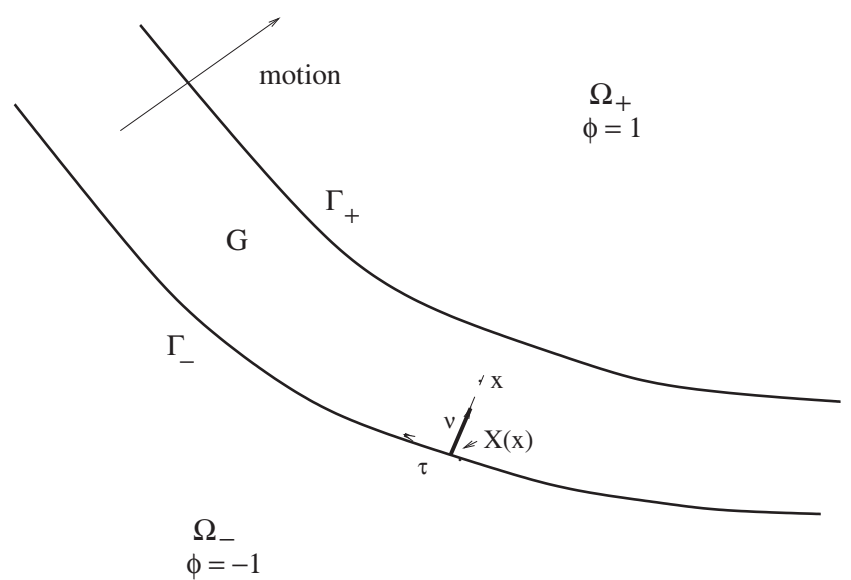

FIG. 1. Configuration of the grain boundary. The coordinate $r$ is the distance from $x$ to $X(x)$ and $s$ is arclength along $\Gamma_{-}$.

the second coming from (2.2). Recall that the function $\phi$ is extended continuously outside $G$ with $\phi(x, t)= \pm 1$ for $x \in \Gamma_{ \pm}(t) \cup \Omega_{ \pm}(t)$.

For the time being, we disregard the possible intersections of $G$ with the boundary of the domain occupied by the metal specimen. Those intersections will generate additional pieces of the boundary of $G$. They will be dealt with in Sections 2.5 and 3 .

We consider only motions such that $G$ moves into $\Omega_{+}(t)$, i.e. $\Gamma_{+}$is the leading edge and $\Gamma_{-}$ the trailing edge. As mentioned above, we have $c(x, t)=\hat{c}(x, t)=0$ in $\Omega_{+}$, and since $D=0$ in $\Omega_{ \pm}$, we have from (2.11) that $c$ is independent of $t$ in $\Omega_{-}$. It may, of course, depend on $x$. In fact, for $x \in \Omega_{-}(t), c(x, t)=c(x, T(x))$, where $T(x)$ is the time at which $x \in \Gamma_{-}(T)$. This is the concentration left behind after the grain boundary has passed the point $x$.

\subsection{Coordinates tied to $G$ (Fig. 1)}

We start with a dimensionless curvilinear coordinate system to be used in $G(0)$. We call it $(r(x, 0)$, $s(x, 0))$, or just $(r, s)$ for short. We denote by $X(x)$ the closest point on $\Gamma_{-}(0)$ to $x$, and define $r(x, 0)$ to be the distance from $x$ to $X(x)$. Let $\nu(x)$ denote the unit vector directed from $X(x)$ toward $x$, and $\tau(x)$ the one obtained by rotating $\nu(x)$ through the angle $\pi / 2$. Let $s(x, 0)$ be signed arclength from some reference point $X_{0}$ on $\Gamma_{-}(0)$ to $X(x), s$ increasing in the direction of $\tau$. We assume the transformation $x \rightarrow(r, s)$ is smooth and invertible. This will be true if $G(0)$ is thin enough.

We shall need to rewrite the equations (2.10), (2.11) within $G(t)$ in terms of scaled curvilinear spatial coordinates. We do this first at $t=0$. Define

$$
z=\frac{r}{\epsilon}, \quad s^{*}=\rho^{-1 / 2} s,
$$

and new functions

$$
\Phi(z, s, 0)=\phi(x, 0), \quad U\left(z, s^{*}, 0\right)=c(x, 0), \quad W\left(z, s^{*}, 0\right)=w(x, 0) .
$$


Here the coordinate $x$ on the right sides is to be expressed as $x(z, s, 0)$ or $x\left(z, s^{*}, 0\right)$ by means of the transformations given above.

An expression (D.6) for $\nabla \cdot D \nabla w$ in this curvilinear system is derived in Appendix D. That represents the left side of (2.11) at $t=0$.

For the right side of (2.11) and left side of (2.10), we must let the coordinate system evolve.

For this purpose, let $Y$ be a curve in space through the point $X_{0}$ which for each $t$ is orthogonal to $\Gamma_{-}(t)$ at the place where they intersect. We define $r(x, t)$ again to be the distance from $x$ to $\Gamma_{-}(t)$, the point on the latter which is closest to $x$ being denoted by $X(x, t)$. We define $\boldsymbol{\nu}(x, t)$ and $\boldsymbol{\tau}(x, t)$ in the same way we did at $t=0$, and $s(x, t)$ to be the signed arclength on $\Gamma_{-}(t)$ from its intersection point with $Y$ to $X(x, t), s$ increasing as we move in the direction $\tau$.

At $t=0$, we have by the chain rule

$$
\phi_{t}(x, 0)=\Phi_{t}(z, s, 0)+\epsilon^{-1} r_{t}(x, 0) \Phi_{z}(z, s, 0)+s_{t}(x, 0) \Phi_{s}(z, s, 0),
$$

where the symbols $x$ on the right are meant to denote $x(z, s, 0)$. Now $r_{t}(x, 0)=-v(s(x, 0), 0)$, where $v(s, 0)$ is the normal velocity of $\Gamma_{-}(0)$ at the point with coordinate $s$.

We may also characterize $s_{t}(x, 0)$ in terms of more familiar things. It is shown, again in Appendix D, that

$$
s_{t}(x, t)=\rho^{-1} S(s, t),
$$

where

$$
S(s, t)=\int_{0}^{s} v^{*}\left(\rho^{-1 / 2} s^{\prime}, t\right) \kappa\left(s^{\prime}, t\right) \mathrm{d} s^{\prime}
$$

and

$$
v^{*}\left(s^{*}, t\right)=\rho v(s, t) .
$$

We therefore have

$$
\phi_{t}(x, 0)=\Phi_{t}(z, s, 0)-\epsilon^{-1} v(s, 0) \Phi_{z}(z, s, 0)+\rho^{-1} S(s, 0) \Phi_{s}(z, s, 0),
$$

with a similar expression for $c_{t}(x, 0)$.

This, together with (D.6), provides the transformation of equations (2.11), (2.12) at time 0 . But at any other time, we could simply take that other time as the time origin and apply the same reasoning to obtain the same formula. Our definition of the evolution of the coordinate system was designed not to depend on the time origin. We therefore define $\Phi(z, s, t), U\left(z, s^{*}, t\right), W\left(z, s^{*}, t\right)$ the same way as in (2.18), and $v(s, t)$ as the velocity of $\Gamma_{-}(t)$, and obtain that (2.10), (2.11) are transformed into

$$
\begin{aligned}
& \rho \epsilon^{2} \Phi_{t}(z, s, t)-\rho \epsilon v(s, t) \Phi_{z}(z, s, t)+\epsilon^{2} S(s, t) \Phi_{s}(z, s, t) \\
& \quad=\Phi+\left(\Phi_{z}\right)_{z}-\frac{\epsilon \kappa}{1-\epsilon z \kappa} \Phi_{z}+\epsilon^{2} \frac{1}{1-\epsilon z \kappa} \partial_{s}\left(\frac{\Phi_{s}}{1-\epsilon z \kappa}\right)-\epsilon p_{\phi}, \\
& \epsilon U_{t}\left(z, s^{*}, t\right)-v(s, t) U_{z}\left(z, s^{*}, t\right)+\epsilon \rho^{-3 / 2} S(s, t) U_{s^{*}}\left(z, s^{*}, t\right) \\
& \quad=\epsilon^{-2}\left(D W_{z}\right)_{z}-\frac{\epsilon^{-1} \kappa}{1-\epsilon z \kappa} D W_{z}+\frac{\rho^{-1}}{1-\epsilon z \kappa} \partial_{s^{*}}\left(\frac{D W_{s^{*}}}{1-\epsilon z \kappa}\right) .
\end{aligned}
$$


These equations hold in $G(t)$. We must add to them the relation (2.12):

$$
W=U+\frac{\hat{\epsilon} \beta}{4}(1+\Phi)^{2} U
$$

and possible boundary conditions on the boundaries of $G(t)$. For $\Phi$, we have (2.16):

$$
\Phi= \pm 1, \quad \nabla \Phi=0 \quad \text { on } \quad \Gamma_{ \pm}(t) .
$$

Consider now the issue of boundary conditions for $U$ (or $c$ ). This is a crucial question in our analysis; the vanishing of $D$ outside $G$ induces a degeneracy in (2.25) which calls into question the regularity and even the definition of solutions. The most extreme case of this irregularity is when $D$ is discontinuous at $\Gamma_{ \pm}$, jumping from a positive value in $G$ to 0 outside. Whatever the concept of solution used, by conservation of solute the normal component of the material flux must be continuous at $\Gamma_{ \pm}$, when measured relative to those two moving curves.

We approach the question of side conditions for $U$ by first describing the physical picture. Since $c=0$ in the parent grain $\left(\Omega_{+}\right)$, there is no flux of material through $\Gamma_{+}$(again, in a frame moving with $\Gamma_{+}$). However, there will be material flux out of $G$ through $\Gamma_{-}$; the latter is deposited in the daughter grain $\Omega_{-}$. This latter flux is generated by diffusion of material along the grain boundary. It is not known a priori, and in fact will only be determined after solving the free-boundary problem which we shall derive in Section 2.4.

Similarly, the value of $c=U$ at the trailing edge is not known at this stage. Therefore, the most conventional boundary conditions for $U$ on $\Gamma$ are not appropriate.

What turns out to be a suitable side condition for $U$, in place of the traditional ones at $\Gamma_{-}$, is that it be the limit of a sequence of solutions of similar problems with nondegenerate diffusions. The functions $U_{n}$ in the sequence should be uniformly bounded and have uniformly bounded fluxes through $\Gamma_{-}$, and this smoothness with respect to the variables $s$ and $t$ should be uniform in $n$.

This characterization of $U$ is studied in great detail in Appendix B, and is used in Sections 2.3.2 and 2.4. For example, Theorem 4 in that appendix shows that the existence of a bounded sequence of solutions $U_{n}$ of (2.25) and (2.12) whose nondegenerate diffusions $D_{n}$ approach the actual degenerate diffusion, and which enjoy the uniformity assumptions given above, ensures that a subsequence converges to a solution $U$.

To summarize, we impose the following side conditions on $c=U$ :

$$
\text { Material flux through } \Gamma_{+}=0 \text {, }
$$

and, in a sense to be spelled out more precisely later (Section 2.3.2),

$U$ is the limit of a sequence of nondegenerate solutions.

It will be shown that a consequence of $\hat{\epsilon} \ll 1$ is that the concentration is nearly uniform across the grain boundary except for a possible sharp change near $\Gamma_{+}$. It was argued in Appendix A of [4], also characterizing it as the limit of nondegenerate solutions, that the concentration should be continuous on the trailing edge, whether or not $D$ is continuous there, and that property, as we shall see, follows from (2.29), so that it holds for our solutions.

The initial-value problem for (2.24), (2.25) would require conditions at $t=0$ to be imposed as well. However, we do not consider the general initial-value problem; rather we use internal layer asymptotics to construct solutions for which $\Phi$ makes a transition from -1 to 1 within the narrow boundary region. Viewed on a macroscopic scale, the boundary regions will reduce to a curve and the solutions will be discontinuous there. 


\subsection{Structure of the solution inside $G(t)$ obtained by asymptotics}

The use of formal asymptotics to solve otherwise difficult problems nearly always proceeds on the basis of assumed (explicitly or implicitly) properties of the solution being sought; the perceived consistency of the asymptotic procedure then lends credence to the existence of a solution with those properties.

In the present case, the basic assumptions are

I. $\kappa(s, t)$ depends in a regular fashion on $(s, t)$, in the sense that it and its first two derivatives with respect to $s$ or $t$ are $\leqslant \mathrm{O}(1)$ quantities. Also $\int_{0}^{s}\left|\kappa\left(s^{\prime}, t\right)\right| \mathrm{d} s^{\prime} \leqslant \mathrm{O}(1)$ for all $s$.

II. $U\left(z, s^{*}, t\right)$ and $W\left(z, s^{*}, t\right)$ depend in a regular fashion on $\left(s^{*}, t\right)$.

III. $\Phi(z, s, t ; \epsilon)$ depends in a regular fashion on $(z, s, t, \epsilon)$ near $\epsilon=0$.

IV. Recalling (2.22)

$$
v(s, t)=\rho^{-1} v^{*}\left(s^{*}, t\right),
$$

we assume that

$$
\begin{aligned}
0<v^{*}\left(s^{*}, t\right) & \leqslant \mathrm{O}(1), \\
\left|v_{s^{*}}^{*}\right|, \quad\left|v_{s^{*} s^{*}}^{*}\right| & \leqslant \mathrm{O}(1) .
\end{aligned}
$$

Of course, all solutions depend on parameters such as $\epsilon$ and $\rho$, but we have not generally indicated that dependence explicitly. Item III above is an exception; dependence of $\Phi$ on $\epsilon$ was shown because the analysis in Section 2.3.1 requires some regularity in that dependence.

Some interpretation of these scalings is called for. In terms of the dimensional quantities given in (2.9), the implication of I and III is that, for the solutions we construct, the shape of the grain boundary will have characteristic radius of curvature $\frac{\delta}{\epsilon}$ or larger. The implication of II, however, is that the variation of $c$ along the grain boundary will typically occur with characteristic length $\frac{\delta}{\epsilon} \rho^{1 / 2}=\sqrt{\frac{D_{m} \tau}{\epsilon}}$ or larger. The integral condition in I follows from the first part of I if the length of $\Gamma_{-}$is $\leqslant \mathrm{O}(1)$. In the case of the steady motion studied in Section 3, when the length of $\Gamma$ may be infinite, it is also satisfied.

Finally, IV (2.30) implies that the characteristic velocity of the grain boundary will be at most $\frac{\epsilon \delta}{\tau}$. However, we note here that some solutions, such as some trailing configurations considered in Section 3 with small $\rho$, are such that the characteristic length of the grain boundary shape, as well as that of the variation of $c$ along it, are both $\frac{\delta}{\epsilon}$, and the characteristic velocity is $\frac{\epsilon^{2} D_{m}}{\delta}$.

In view of the comments following (2.29), we cannot expect $U\left(z, s^{*}, t ; \epsilon\right)$ and $W\left(z, s^{*}, t ; \epsilon\right)$ to be regular in $\epsilon$ uniformly up to $\Gamma_{+}$; as will be indicated in Section 2.3.2, there will generally be a thin region near $\Gamma_{+}$wherein they may be significantly different from their values elsewhere. This exception is allowed for in assumption II above, which claims nothing about $z$-dependence.

\subsubsection{The phase function. We write (2.24) in the form}

$$
\Phi_{z z}+\Phi=\epsilon\left[-v^{*} \Phi_{z}+\kappa \Phi_{z}+p_{\phi}\right]+\mathrm{O}\left(\epsilon^{2}\right) .
$$

This equation is to hold in the region $G$ defined by (2.15), i.e. $-1<\Phi<1$, on the boundary of which, by (2.27),

$$
\Phi= \pm 1 \quad \text { and } \quad \Phi_{z}=0
$$


This problem has the unusual feature that the domain of the unknown function $\Phi$ depends on $\Phi$ itself; that feature results from the double-obstacle nature of equations (2.1), (2.2) for $\phi$. In effect, we have a free-boundary problem for the determination of the boundaries of $G$ with reference to the stretched coordinates (our eventual aim is to derive a quite different free-boundary problem: namely, one for the determination of $G(t)$ with reference to the original coordinates $(x, t))$.

A simple expansion in powers of $\epsilon$ is therefore inappropriate as an approximation scheme. Instead, we shall employ an $\epsilon$-dependent change of variable (to be determined)

$$
\zeta=(1+\alpha \epsilon) z
$$

and find a solution of (2.33) in the form

$$
\Phi(z, s, t)=\Phi^{0}(\zeta, s, t)+\epsilon \Phi^{1}(\zeta, s, t) .
$$

The task will be to find $\Phi^{0}, \Phi^{1}$ and the constant $\alpha$.

Substituting (2.36) into (2.33), we find

$$
(1+\alpha \epsilon)^{2}\left(\Phi_{\zeta \zeta}^{0}+\epsilon \Phi_{\zeta \zeta}^{1}\right)+\Phi^{0}+\epsilon \Phi^{1}=\epsilon\left[\left(-v^{*}+\kappa\right) \Phi_{\zeta}^{0}+p_{\phi}\right]+\mathrm{O}\left(\epsilon^{2}\right) .
$$

Expanding in $\epsilon$, we obtain

$$
\Phi_{\zeta \zeta}^{0}+\Phi^{0}=0
$$

The only monotone solution of this equation satisfying the boundary requirements (2.34) is

$$
\Phi^{0}(\zeta, s, t)=-\cos \zeta, \quad 0<\zeta<\pi .
$$

(It is natural to select the monotone solution; this simply reflects a gradual change in the structure of the material from that associated with one grain to that of the other.)

From this, we conclude that the trailing and leading edges are characterized by $\zeta=0, \pi$, respectively. In terms of the distance variable $r$, we obtain that the nondimensional thickness of the grain boundary is $\pi \epsilon$ to leading order. From (2.9), we therefore see that the dimensional grain boundary thickness is $\pi \delta$, corroborating the claim made earlier that $\delta$ is a constant comparable to that thickness. An $\mathrm{O}(\epsilon)$ approximation to the thickness which follows from (2.35) is $\pi \epsilon(1+\alpha \epsilon)$, where $\alpha$ will be obtained shortly.

The $\mathrm{O}(\epsilon)$ part of (2.37) is

$$
\Phi_{\zeta \zeta}^{1}+\Phi^{1}=\left[-\left(v^{*}-\kappa\right) \sin \zeta+p_{\phi}\right]-2 \alpha \cos \zeta .
$$

Since the boundary of the domain of $\Phi$ is now set at $\zeta=0, \pi$, we must impose the following boundary conditions on the solution of (2.40), which follow from (2.27):

$$
\begin{array}{lll}
\Phi^{1}=0 & \text { at } \quad \zeta=0, \pi, \\
\Phi_{\zeta}^{1}=0 & \text { at } \quad \zeta=0, \pi .
\end{array}
$$

This constitutes four conditions, although (2.40) is only a second-order equation. In addition, an orthogonality condition on the right side of (2.40) is needed for the existence of a solution satisfying (2.41). Altogether, this makes three extra conditions to be satisfied. They can be fulfilled 
by (1) choosing $v$ so that the solvability condition is satisfied, (2) choosing $\alpha$ appropriately, and (3) noting that any solution of (2.40) and (2.41) may be supplemented by a term $\gamma \sin \zeta$, for arbitrary $\gamma$, thus affecting the boundary values of $\Phi_{\zeta}^{1}$. One then chooses $\gamma$ appropriately.

Consider first the boundary value problem (2.40) under only the Dirichlet conditions, i.e. (2.41). The operator on the left is singular, having $\sin \zeta$ as nullfunction. Therefore, for solvability, the right side must be $L^{2}$-orthogonal to $\sin \zeta$. From (2.5) and (2.7) we have

$$
p_{\phi}=\frac{\pi}{4}\left(1+\Phi^{0}\right) U^{2}+\mathrm{O}(\epsilon) .
$$

From this, (2.39), and the fact that $z=\zeta+\mathrm{O}(\epsilon)$, the orthogonality condition assumes the form

$$
v^{*}\left(s^{*}, t\right)=\kappa(s, t)+\frac{1}{2} \int_{0}^{\pi} U\left(z, s^{*}, t\right)^{2} \sin z(1-\cos z) \mathrm{d} z+\mathrm{O}(\epsilon),
$$

since the last term in (2.40) is already orthogonal to $\sin \zeta$. This provides an enhanced motion by curvature law in leading order. It will appear later in the simpler form (2.74).

Once the condition (2.44) is satisfied, the Dirichlet problem (2.40), (2.41) can be solved in the form

$$
\Phi^{1}=\Phi^{\dagger}+\alpha \Phi^{*}+\gamma \sin \zeta
$$

where $\Phi^{\dagger}$ is a particular solution of (2.40), (2.41) with $\alpha=0, \Phi^{*}$ is such a particular solution with the right side of (2.40) replaced by $-2 \cos \zeta$, and $\gamma$ is an arbitrary (for the moment) constant reflecting the fact that any solution of the Dirichlet problem may be supplemented by a nullfunction. One immediately determines $\Phi^{*}=\zeta \sin \zeta$.

Our last task is to choose the remaining unknown constants $\gamma$ and $\alpha$ so that (2.42) is satisfied. The vanishing of the $\zeta$-derivative at the two points $\zeta=0, \pi$ results in the two equations

$$
\Phi_{\zeta}^{\dagger}(0)+\gamma=0, \quad \Phi_{\zeta}^{\dagger}(\pi)-\alpha \pi-\gamma=0 .
$$

These two equations have a unique solution $(\alpha, \gamma)$. This completes our construction of the solution (2.36) of (2.33).

We shall need the following property of the exact solution $\Phi(z, s, t)$ : there exist positive constants $a_{1}, a_{2}$ such that for small $z>0$,

$$
a_{1} z^{2} \leqslant \Phi(z, s, t)+1 \leqslant a_{2} z^{2} .
$$

To see this, note that by (2.39) the dominant term $\Phi^{0}+1$ in the expansion of $\Phi+1$ is $\mathrm{O}\left(z^{2}\right)$ and is bounded below by $\frac{1}{4} z^{2}$ for small $z$, and the boundary conditions (2.34) imply that all higher terms $\Phi^{\ell}$ in the expansion satisfy $\Phi^{\ell}=\mathrm{O}\left(z^{2}\right)$. This last argument applies also to tangential derivatives, giving us

$$
\left|\Phi_{s s}(z, s, t)\right|+\left|\Phi_{s}(z, s, t)\right| \leqslant a_{2} z^{2} .
$$

The same is true near the other boundary $\Gamma^{+}$of $G$, which is given (say) by $z=z_{1}(s, t)$. We have, for small $\eta>0$,

$$
a_{1} \eta^{2} \leqslant 1-\Phi\left(z_{1}-\eta, s, t\right) \leqslant a_{2} \eta^{2} .
$$


Combining (2.46) and (2.6), we have

$$
a_{3} z^{2 v}<D(\Phi(z, s, t)) \leqslant a_{4} z^{2 v}
$$

for small $z>0$, and $D(\Phi)$ is monotonic in $z$ for $z$ near 0 ; moreover, the analogous results hold for $z$ near $z_{1}$.

Finally, because of (2.46) and (2.36) we may write $\Phi(z, s, t, \epsilon)=\Phi_{0}(z)+z^{2} \epsilon \Psi(z, s, t, \epsilon)$ with regular $\Psi$, so that

$$
\partial_{s} D(\Phi(z, s, t))=D^{\prime}(\Phi) \partial_{s} \Phi=\epsilon D^{\prime}(\Phi) z^{2} \partial_{s} \Psi
$$

and hence

$$
\partial_{s} D(\Phi(z, s, t))=\epsilon h(z, s, t) D(\Phi(z, s, t)),
$$

where $h=D^{-1} D^{\prime} z^{2} \partial_{s} \Psi$. We show that $h$ is bounded. This is only an issue for $z$ near 0 or $z_{1}$. In the former case (the latter is similar), we know from (2.6) that

$$
D(\Phi)^{-1} \leqslant C(\Phi+1)^{-v}, \quad D^{\prime}(\Phi) \leqslant C(\Phi+1)^{\nu-1} .
$$

This, with (2.46), indicates

$$
|h| \leqslant C(\Phi+1)^{-1} z^{2} \leqslant C .
$$

2.3.2 The concentration. As indicated before in assumption II, we assume that $U$ and $W$ depend in a regular fashion on $\left(s^{*}, t\right)$. The character of their dependence on $z$ and $\epsilon$, however, requires a very careful derivation.

We may rewrite (2.25) as (with $D=D(\Phi(z, s, t))$

$$
\begin{aligned}
\partial_{z}\left(D \partial_{z} W\right)= & \epsilon \kappa D \partial_{z} W\left(1+\epsilon q_{2}(z, s, t, \epsilon)\right) \\
& -\epsilon^{2} \rho^{-1}\left[\partial_{s^{*}}\left(\left(1+\epsilon q_{4}(z, s, t, \epsilon)\right) D \partial_{s^{*}} W\right)\left(1+\epsilon q_{3}(z, s, t, \epsilon)\right)+v^{*}\left(s^{*}, t\right) \partial_{z} U\right] \\
& +\epsilon^{3}\left(\partial_{t} U+\rho^{-3 / 2} U_{s^{*}} S\left(s^{*}, t\right)\right)
\end{aligned}
$$

for some bounded regular functions $q_{2}, q_{3}, q_{4}$ which can be readily obtained in terms of $\kappa$ from (2.25). They and their derivatives are bounded independently of $\epsilon$ because of our assumption I that $\kappa$ and its $s$-derivatives are so bounded. (Notice that $D$ and $q_{i}$ depend on $s$, but $W$ and $v^{*}$ are considered to be functions of $s^{*}=\rho^{-1 / 2} s$.)

We shall obtain the desired estimates for $W$ and $U$ by casting (2.51) in the form of the ODE treated in Theorem 3 of Appendix B. The assumptions of that theorem must be justified. This is delicate because of the degeneracy of $D$ for $z<0$ and $z>z_{1}(s, t)$; in fact, the concept of solution given by (2.29) must be brought into the argument. But first, we make the correlation between (2.51) and (B.1).

The functions $W$ and $U$ are related by (2.26). Hence, since $\Phi$ is smooth in all variables (assumption III),

$$
U=W\left(1+\hat{\epsilon} \beta q_{1}(z, s, t, \epsilon)\right), \quad \partial_{z} U=\partial_{z} W\left(1+\hat{\epsilon} \beta q_{1}\right)+\hat{\epsilon} \beta W \partial_{z} q_{1}
$$


for a function $q_{1}$ with the same boundedness properties as the other $q_{i}$. We make the substitutions indicated by $(2.52)$ in (2.51). For the moment, we freeze the variables $(s, t)$ in $(2.51)$ and set $D(\Phi(z))=d(z)$. As shown following (2.49), this function is monotone for $z$ near 0 or $z_{1}$. In (B.1) we set

$$
\begin{gathered}
\sigma_{1}=\epsilon^{2} \rho^{-1} v^{*}, \quad \sigma_{2}=\epsilon, \quad \sigma_{3}=\epsilon^{2} \rho^{-1}, \quad \sigma_{4}=\epsilon^{3} \rho^{-3 / 2}, \quad \sigma_{5}=\hat{\epsilon} \beta, \\
f_{1}=q_{1}, \quad f_{2}=-\kappa\left(1+\epsilon q_{2}\right), \quad d(z) f_{3}=-\partial_{s^{*}}\left(d(z)\left(1+\epsilon q_{4}\right) \partial_{s^{*}} W\right)\left(1+\epsilon q_{3}\right), \\
f_{4}=\rho^{3 / 2} \partial_{t} U+S \partial_{s^{*}} W .
\end{gathered}
$$

Then (2.51) becomes (B.1).

Now consider the justification of the hypotheses of Theorem 3. The boundedness of $f_{1}$ and $f_{2}$ follows from that of the $q$, and that of $f_{4}$ from assumptions I-IV in Section 2.3 and (D.12). That of $f_{3}$ is considered below.

One of the hypotheses is the continuity of $W$ at $z=0$. For this we use our basic stipulation (2.29), along with Theorem 4. Suppressing dependence on $(s, t)$, we express the diffusivity $D(\Phi(z))=d(z)$ as the limit of a sequence of strictly positive smooth functions $d_{n}(z)$ defined in an expanded interval $\left(-a, z_{1}\right)$ for some $a>0$. We then posit that $W$ is the limit of a sequence of solutions $W_{n}$ of (2.51) with $D$ replaced by $d_{n}(z)$. According to Theorem 4, the existence of such a limit $W$, uniform in closed intervals excluding $z_{1}$, its continuity at $z=0$, and the fact that its diffusive flux $J=\lim _{z \downarrow 0} d(z) \partial_{z} W(z)=0$, follows merely from the assumed existence of a sequence of solutions $W_{n}$ (with $d=d_{n}$ ) which are smooth in $\left(s^{*}, t\right)$ uniformly in $n$ and whose diffusive fluxes $J_{n}=d_{n} \partial_{z} W_{n}$ at $z=-a$ are uniformly bounded. (By smooth in $\left(s^{*}, t\right)$, it will suffice to assume that three derivatives with respect to $s^{*}$ and two with respect to $t$ are uniformly bounded.) The condition (2.29) is to be interpreted that $W$ is a limit in the sense of Theorem 4 .

Being a uniform limit near $z=0$, the solution $W$ is regular there in $z$ as well as in $\left(s^{*}, t\right)$, but not necessarily so near $z=z_{1}$.

The only other hypotheses of Theorem 3 to check are (B.3) and that $f_{3}=-d(z)^{-1}[d(z)(1+$ $\left.\epsilon q_{4}\right) \partial_{s^{*}}^{2} W+\left(\partial_{s^{*}}\left(d(z)\left(1+\epsilon q_{4}\right)\right) \partial_{s^{*}} W\right]\left(1+\epsilon q_{3}\right)=-\partial_{s^{*}}^{2} W\left(1+\epsilon q_{3}\right)\left(1+\epsilon q_{4}\right)-d(z)^{-1} \partial_{s^{*}}(d(z)(1+$ $\left.\left.\epsilon q_{4}\right)\right) \partial_{s^{*}} W\left(1+\epsilon q_{3}\right)$ is bounded. This is where we use (2.50). It implies that $d(z)^{-1} \partial_{s} d(z)$ is bounded, and so is $d(z)^{-1} \partial_{s^{*}} d(z)$, since $\partial_{s^{*}}=\rho^{1 / 2} \partial_{s}$ and $\rho \leqslant \mathrm{O}(1)$. The boundedness of $\partial_{s^{*}} W$ and $\partial_{s^{*}}^{2} W$ comes from assumption II. Assumption (B.3) follows from (2.50). Therefore, all the hypotheses of Theorem 3 are met.

Theorem 3 provides estimates, depending on $\epsilon$, associated with $W$, and they will now be used.

It is remarkable that the needed properties following from Theorem 3 depend crucially on the positivity of the velocity factor $v^{*}$ in the higher-order term $-\epsilon^{2} \rho^{-1} v^{*} \partial_{z} U$ in (2.51).

We restrict $v^{*}$ only by (2.31) and seek estimates which depend explicitly on $v^{*}$. Since by (2.14) and (2.53)

$$
\sigma_{2}+\sigma_{3}+\sigma_{5}+\frac{\sigma_{4}}{\sigma_{1}} \leqslant C\left(\hat{\epsilon} \beta+\frac{\epsilon^{2}}{\rho}+\frac{\epsilon \rho^{-1 / 2}}{v^{*}}\right) \leqslant C\left(\hat{\epsilon} \beta+\frac{\epsilon \rho^{-1 / 2}}{v^{*}}\right),
$$

the conclusion (B.2) of Theorem 3 (recall $J=0$ ) implies (for each $(s, t)$ )

$$
|W(z)-W(0)| \leqslant C\left(\hat{\epsilon} \beta+\frac{\epsilon \rho^{-1 / 2}}{v^{*}}\right)
$$

for $z \leqslant \pi / 2$. 
Moreover, (B.4) $(k=2)$ provides the further estimate

$$
d(z)\left|W_{s^{*} s^{*}}(z)-W_{s^{*} s^{*}}\left(\frac{\pi}{2}\right)\right| \leqslant C \epsilon^{2} \rho^{-1}
$$

for all $z$, and by (2.26) and (B.4) with $(k=0)$,

$$
d(z)\left|U(z)-U\left(\frac{\pi}{2}\right)\right| \leqslant C\left(\hat{\epsilon} \beta+\epsilon^{2} \rho^{-1}\right) .
$$

From (2.26) and (2.55), we get

$$
|U(z)-U(0)| \leqslant C\left(\hat{\epsilon} \beta+\frac{\epsilon \rho^{-1 / 2}}{v^{*}}\right),
$$

and hence in particular

$$
v^{*}\left|U(0)-U\left(\frac{\pi}{2}\right)\right| \leqslant C\left(\hat{\epsilon} \beta+\epsilon \rho^{-1 / 2}\right) .
$$

\subsection{The motion of $G$ : the developed case to lowest order}

By 'developed' we mean that transients operating on a faster time scale, as explained below, have disappeared.

Fix $s_{1}^{*}<s_{2}^{*}$, where these are $\mathrm{O}(1)$ numbers. We consider only the portion of $G$ given by

$$
G^{*}(t)=G(t) \cap\left\{\rho^{1 / 2} s_{1}^{*}<s<\rho^{1 / 2} s_{2}^{*}\right\} .
$$

Set

$$
M(t)=\int_{G^{*}(t)} c(x, t) \mathrm{d} x .
$$

This is the total mass of solute contained in $G^{*}$. We, of course, continue to seek solutions satisfying II. In particular, in the coordinate system attached to the moving strip $G(t)$, the time rate of change of $c$ and $c^{*}$ is at most an $\mathrm{O}(1)$ quantity. We interpret this as saying that any fast transients have disappeared. Also, the $s^{*}$-derivatives of $U$ and $W$ are bounded. Since the size of $G^{*}$ (in reference to the variable $x$ in (2.9)) is $\mathrm{O}\left(\epsilon \rho^{1 / 2}\right)$, we have

$$
M,\left|\frac{\mathrm{d} M}{\mathrm{~d} t}\right| \leqslant C \epsilon \rho^{1 / 2},
$$

where $C$ is independent of $\epsilon$ and $\rho$.

We now write an equation for the conservation of mass $M(t)$ in $G^{*}$, and derive one of our freeboundary equations as an asymptotic approximation to it. We do this for time $t=0$, but the result is valid for any $t$. The mass changes only due to the flux of solute through the boundary of $G^{*}$, which consists of four parts: $\Gamma_{-} \cap \partial G^{*}, \Gamma_{+} \cap \partial G^{*}$, and the two ends where $s=\rho^{1 / 2} s_{i}^{*}, i=1,2$. We consider them separately.

Condition (2.28) implies there is no such flux through $\Gamma_{+}$. Consider the trailing edge $\Gamma_{-}$. As we have noted, the characterization (2.29) implies that $J=\left.d(z) \partial_{z} W(z)\right|_{z=0}=0$, which means there 
is no diffusive flux through $\Gamma_{-}$. The convective flux is

$$
\begin{gathered}
F_{1}(0)=-\int_{\Gamma_{-} \cap \partial G^{*}} v c \mathrm{~d} s=-\int_{s_{1}^{*}}^{s_{2}^{*}} \rho^{-1} v^{*}\left(s^{*}, 0\right) U\left(0, s^{*}, 0\right) \rho^{1 / 2} \mathrm{~d} s^{*} \\
=-\rho^{-1 / 2} \int_{s_{1}^{*}}^{s_{2}^{*}} v^{*}\left(s^{*}, 0\right) U\left(0, s^{*}, 0\right) \mathrm{d} s^{*} .
\end{gathered}
$$

This represents flux of material out of $G^{*}$ being deposited in the growing grain. Expression (2.63) relies on $U$ being continuous on $\Gamma$, and this in turn follows from the continuity of $W$ at $z=0$. As we brought out before, this comes from (2.29) via Theorem 4.

From (2.63), (2.59) we find

$$
F_{1}(0)=-\rho^{-1 / 2} \int_{s_{1}^{*}}^{s_{2}^{*}} v^{*}\left(s^{*}, 0\right) U\left(\frac{\pi}{2}, s^{*},\right) \mathrm{d} s^{*}+\mathrm{O}\left(\hat{\epsilon} \beta \rho^{-1 / 2}+\epsilon \rho^{-1}\right)=\rho^{-1 / 2} \mathrm{O}\left(\hat{\epsilon} \beta+\epsilon \rho^{-1 / 2}\right) .
$$

Next, we consider the flux through the ends $\left\{s=\rho^{1 / 2} s_{i}^{*}\right\}$ of $G^{*}$. It is given in terms of the internal material flux vector $\boldsymbol{J}(r, s, t)$ as

$$
F_{2}=\left[\int_{\left\{s=\rho^{1 / 2} s_{1}^{*}\right\} \cap \partial G^{*}}-\int_{\left\{s=\rho^{1 / 2} s_{2}^{*}\right\} \cap \partial G^{*}}\right] \boldsymbol{J}(r, s, 0) \cdot \boldsymbol{\tau}(r, s, 0) \mathrm{d} r,
$$

where $\tau$ is the unit vector normal to the boundary of $G^{*}$ at the ends pointing in the direction of increasing $s$. Since the two ends are lines of constant $s$, the normal derivative is in the direction of the $s$-derivative, and in fact $\tau \cdot \nabla w=\frac{\partial w}{\partial s}=\rho^{-1 / 2} \frac{\partial W}{\partial s^{*}}$.

From (2.11) we know that

$$
\boldsymbol{J}=-\epsilon^{-1} D(\phi) \nabla w .
$$

On $\left\{s^{*}=s_{i}^{*}\right\} \cap \partial G^{*}$, we have $\boldsymbol{J}=-\epsilon^{-1} \rho^{-1 / 2} D W_{s^{*}}$, and since $\mathrm{d} r=\epsilon \mathrm{d} z$ and $z_{1}=\pi+\mathrm{O}(\epsilon)$,

$$
F_{2}=\left[\int_{s^{*}=s_{1}^{*}}-\int_{s^{*}=s_{2}^{*}}\right] \rho^{-1 / 2} D W_{s^{*}} \mathrm{~d} z=\int_{0}^{\pi} \int_{s_{1}^{*}}^{s_{2}^{*}} \rho^{-1 / 2}\left(D W_{s^{*}}\right)_{s^{*}} \mathrm{~d} s^{*} \mathrm{~d} z(1+\mathrm{O}(\epsilon)) .
$$

Now by (2.50), $\left(D W_{s^{*}}^{*}\right)_{s^{*}}=\left(\partial_{s^{*}} D\right) \partial_{s^{*}} W+D \partial_{s^{*}}^{2} W=\rho^{1 / 2} \partial_{s} D \partial_{s^{*}} W+D \partial_{s^{*}}^{2} W=\rho^{1 / 2} \epsilon h D \partial_{s^{*}} W+$ $D \partial_{s^{*}}^{2} W$. By (B.4) $(k=2)$ we have that $D(\Phi(z, s))\left[\partial_{s^{*}}^{2} W(z, s)-\partial_{s^{*}}^{2} W(\pi / 2, s)\right]=\mathrm{O}\left(\epsilon^{2} \rho^{-1}\right)$, so that $\left(D W_{s^{*}}^{*}\right)_{s^{*}}=D \partial_{s^{*}}^{2} W\left(\pi / 2, s^{*}, t\right)+\mathrm{O}\left(\epsilon^{2} \rho^{-1}+\epsilon \rho^{1 / 2}\right)$. Therefore

$$
F_{2}=\rho^{-1 / 2} \int_{s_{1}^{*}}^{s_{2}^{*}} \int_{0}^{\pi} D(\Phi(z, s)) \mathrm{d} z \partial_{s^{*}}^{2} W\left(\pi / 2, s^{*}, t\right) \mathrm{d} s^{*}+\mathrm{O}\left(\epsilon^{2} \rho^{-3 / 2}+\epsilon\right) .
$$

We now use (2.26), (2.14) and $\partial_{s^{*}}^{2} W\left(\pi / 2, s^{*}, t\right)=\partial_{s^{*}}^{2} U\left(\pi / 2, s^{*}, t\right)+\mathrm{O}(\hat{\epsilon} \beta)$ to express this as

$$
\begin{aligned}
F_{2}= & \pi D_{0} \rho^{-1 / 2} \int_{s_{1}^{*}}^{s_{2}^{*}} \partial_{s^{*}}^{2} U\left(\pi / 2, s^{*}, t\right) \mathrm{d} s^{*}+\mathrm{O}\left(\hat{\epsilon} \beta \rho^{-1 / 2}+\epsilon^{2} \rho^{-3 / 2}+\epsilon\right) \\
& =\cdots+\rho^{-1 / 2} \mathrm{O}\left(\hat{\epsilon} \beta+\frac{\epsilon^{2}}{\rho}\right)
\end{aligned}
$$


where $D_{0}=\frac{1}{\pi} \int_{0}^{\pi} D(-\cos \zeta) \mathrm{d} \zeta$.

Our conservation equation is

$$
F_{1}+F_{2}=\frac{\mathrm{d}}{\mathrm{d} t} M(0)
$$

From (2.62), (2.64), (2.68), (2.14) and the arbitrariness of $s_{i}^{*}$, this implies that at $z=\frac{\pi}{2}$

$$
\pi D_{0} \rho^{-1 / 2} \partial_{S^{*}}^{2} U-\rho^{-1 / 2} v^{*} U=\rho^{-1 / 2} \mathrm{O}\left(\hat{\epsilon} \beta+\epsilon \rho^{-1 / 2}\right) .
$$

Hence

$$
\pi D_{0} \partial_{S^{*}}^{2} U-v^{*} U=\mathrm{O}\left(\hat{\epsilon} \beta+\epsilon \rho^{-1 / 2}\right) .
$$

The error term in (2.70) is $\ll 1$ by (2.14).

At this point we specify the constant $D_{m}$ in (2.7) so that $\pi D_{0}=1$. This means that in terms of the original dimensional diffusivity $\hat{D}(\phi)$,

$$
D_{m}=\int_{0}^{\pi} \hat{D}(-\cos \zeta) \mathrm{d} \zeta,
$$

and $\bar{D}=D_{m} / \pi$ is indeed close to the average diffusivity in the grain boundary.

We shall obtain one of our limiting evolution equations, governing the diffusion of solute along the free boundary, by setting $\pi D_{0}=1$ and neglecting the error term on the right of (2.70):

$$
\partial_{s^{*}}^{2} U\left(\pi / 2, s^{*}, t\right)-v^{*}\left(s^{*}, t\right) U\left(\pi / 2, s^{*}, t\right)=0 .
$$

The second equation for the free-boundary problem comes about by approximating (2.44). Let $g(z)=\sin z(1-\cos z), \quad \eta=(\beta \hat{\epsilon})^{(1 / 2 v)}$ (see (2.6)), and write $\int_{0}^{\pi} U^{2} g(z) \mathrm{d} z=$ $\left[\int_{0}^{\eta}+\int_{\eta}^{\pi-\eta}+\int_{\pi-\eta}^{\pi}\right] U^{2} g(z) \mathrm{d} z$. Observing that $|g(z)|<z$ for small $z$, we estimate the first integral as $\left|\int_{0}^{\eta} U^{2} g \mathrm{~d} z\right| \leqslant C \eta^{2}$. The same estimate holds for the third integral.

For the middle integral, we use (2.57) and (2.49) to estimate, for $\eta<z<\pi-\eta$,

$$
\left|U^{2}\left(z, s^{*}, t\right)-U^{2}\left(\pi / 2, s^{*}, t\right)\right|<C \hat{\epsilon} \beta(d(z))^{-1} \leqslant C \hat{\epsilon} \beta z^{-2 v},
$$

so that

$$
\begin{gathered}
\int_{\eta}^{\pi-\eta}\left|\left(U^{2}\left(z, s^{*}, t\right)-U^{2}\left(\pi / 2, s^{*}, t\right)\right) g(z)\right| \mathrm{d} z \leqslant C \hat{\epsilon} \beta \int_{\eta}^{\pi} z^{1-2 v} \mathrm{~d} z=C \max \left[\hat{\epsilon} \beta \eta^{2-2 v}, \hat{\epsilon} \beta\right] \\
\leqslant C\left(\hat{\epsilon} \beta+(\beta \hat{\epsilon})^{1 / \nu}\right) .
\end{gathered}
$$

Since $\int_{0}^{\pi} g(z) \mathrm{d} z=2$, we have from (2.44)

$$
v^{*}\left(s^{*}, t\right)=\kappa\left(s^{*}, t\right)+U^{2}\left(\pi / 2, s^{*}, t\right)+\mathrm{O}\left(\hat{\epsilon} \beta+(\beta \hat{\epsilon})^{1 / v}\right) .
$$

In this equation and in (2.72) we now revert to the function $v(s, t)=\rho^{-1} v^{*}\left(s^{*}, t\right)(2.30)$ and the variable $s=\rho^{1 / 2} s^{*}$, omit the error terms, and define

$$
u(s, t)=U\left(\pi / 2, s^{*}, t\right) .
$$


Thus $u(s, t)$ represents the concentration on the mid-line through the grain boundary.

The resulting free-boundary problem is

$$
\begin{aligned}
& \rho v=\kappa+u^{2}, \\
& u_{s s}-v u=0 .
\end{aligned}
$$

In these equations, $v(s, t)$ and $\kappa(s, t)$ are velocity and curvature of the curve $\Gamma_{-}(t)$. Since this single curve will serve to represent the grain boundary $G(t)$ in this free-boundary formulation, we rename it $\Gamma(t)$. The function $u(s, t)$ is a concentration associated to the point $s$ on $\Gamma(t)$. We have defined it as the concentration at the mid-line location corresponding to the point $s$, but this detail is unimportant within the free-boundary context. (If $\rho^{1 / 2} v^{*}$ is not small, we see by $(2.58)$ that $u(s, t)$ is also an approximation to the concentration on $\Gamma(t)$ itself, but we wish to allow the possibility that $v^{*}$ be arbitrarily small.)

The first equation (2.74) is a motion by curvature law with a forcing term $u^{2}$. The importance of the curvature $\kappa$ in grain boundary motion was first shown by [1] in a different connection. Rigorous asymptotics for the double-obstacle phase field equation in the form (2.1) with $u$ being prescribed were carried out in [10]; see [9] for a review.

With $\rho>0$, equations (2.74), (2.75), together with the prescription of initial data for $u$ and $\Gamma$, may make a reasonable problem for the evolution of $\Gamma(t)$ and $u(s, t)$, except that there is apparently a severe compatibility constraint which the initial data $(u(s, 0), \Gamma(0))$ must satisfy. Namely, $u(s, 0)$ and $\kappa(s, 0)$ should be such that there exists a function $v(s, 0)$ so that $(2.74),(2.75)$ are satisfied at $t=0$. In other words, at $t=0, u_{s s}-\frac{1}{\rho}\left(\kappa+u^{2}\right) u=0$.

Instead of the initial-value problem, one may instead wish to consider solutions for which $\Gamma(t)$ represents a curve moving with constant speed without changing its shape and $u(s, t)$ is independent of $t$. Such a steady problem is the subject of Section 3 of this paper; see also the comments in Section 2.5.

If $\rho$ is very small, which is the typical case, one is tempted to approximate the problem by setting $\rho=0$ in (2.74). However, it is not likely that the resulting equations specify a well-posed evolution problem. On the other hand, the steady Problem II considered in Section 3 does make sense. This point will be taken up in Section 3.4.

\subsection{Plate problems}

In all this, we have supposed that $G$ does not meet the boundary of the domain $\Omega$. However, in experiments involving metal plates, the grain boundary will typically meet at least one of the plate's faces and terminate there. The point of intersection will itself move along the face. If there is no grooving, the appropriate boundary condition at the intersection, in our phase field model, will be that the normal derivative of $\Phi$ is 0 , and since $\Phi$ is a function of $z$ alone to lowest order (2.39), this translates in the sharp-interface limit into the condition that $\Gamma(t)$ must, for each value of $t$, meet the face orthogonally. If grooving occurs, the face is met at a different angle; see [3] for an analysis of that more general case.

The concentration will also be subject to a boundary condition at the face. If the plate is immersed in a reservoir which acts as a source for solute material, the concentration in that reservoir can (and will) be taken as the characteristic value $c^{\dagger}$, and the boundary condition there for the nondimensional concentration will be $u=1$.

For example, suppose the plate is given as the slab $\left\{-2 H \leqslant x_{2} \leqslant 0\right\}$ in the $x_{1}, x_{2}$ plane, $\Gamma(t)$ connects the faces $\left\{x_{2}=0\right\}$ and $\left\{x_{2}=-2 H\right\}$, and there is such a reservoir outside the plate. Then 
the conditions

$$
\Gamma(t) \text { has a tangent perpendicular to the face at } x_{2}=0,-2 H,
$$

$$
u=1 \quad \text { at } \quad x_{2}=0,-2 H,
$$

must be added to equations (2.74), (2.75).

In the variations discussed below, similar comments can be made about boundary conditions.

\subsection{The full problem, accurate to $\mathrm{O}(\epsilon)$}

A more accurate evolution problem which obviates the stated constraint on the initial conditions given following (2.75) is obtained by keeping the $\mathrm{O}(\epsilon)$ terms in the above analysis. A second advantage of going to next order is that we may relax the assumption $U_{t} \leqslant \mathrm{O}(1)$ used in Section 2.4 , replacing it by $U_{t} \leqslant \mathrm{O}\left(\epsilon^{-1}\right)$. We provide the result in the case that $D(\Phi)$ is a constant in the grain boundary, so that it is discontinuous at $\Gamma_{ \pm}(t)$. This case corresponds to setting $v=0$ in (2.6). Also, we restrict to the case $\rho^{-1}=\mathrm{O}(1)$ and $c^{\dagger}=1$, so that $\epsilon=\hat{\epsilon} / \pi$. In fact, we set $\rho=1$ for simplicity, so that $s^{*}=s$. Because of the normalization (2.71), since $D_{m}=1$, we have $D=\frac{1}{\pi}$. The details of the derivation of the following are given in Appendix $\mathrm{C}$.

Except for errors of the order $\mathrm{O}\left(\epsilon^{2}\right)$, the more accurate evolution problem for $u$, to replace (2.75) and (2.74), is

$$
\rho v=\kappa(1+\epsilon C)+u^{2}\left(1+\epsilon\left(B+A u^{2}\right)\right),
$$

and

$$
\epsilon^{*} u_{t}=\left(u_{s}\left(1+\epsilon u^{2} \alpha\right)\right)_{s}-v u-\epsilon^{*} u v \kappa,
$$

where $\epsilon^{*}=\frac{\epsilon \pi}{1+g_{1} \epsilon}$, and the constants $A, B, C, \alpha, g_{1}$ can be calculated and are independent of $\epsilon$ and $u$.

The pair of equations (2.79), (2.78), together with the arbitrary initial prescription of $\Gamma(0)$ and $u(s, 0)$, constitute a reasonable initial-value problem for the determination of $\Gamma(t)$ and $u(s, t)$. The short time existence of a solution of a case of this initial-value problem was proved by Mayer and Simonett [16], [17].

The significance of the terms on the right side of (2.79) is as follows. The first represents the change in concentration due to diffusion along the grain boundary, the second is the change due to loss of solute atoms to the daughter grain $\Omega_{-}$during the motion of the grain boundary, and the last is the change due to the stretching of the boundary during its motion.

\subsection{The quick-transient problem}

When the prescribed initial data $(u(s, 0), \Gamma(0))$ for (2.79), (2.74) do not satisfy the compatibility constraint given for (2.74), (2.75), there may be an initial rapid process wherein $u$ is adjusted to satisfy the constraint. We give here an approximate description of that process in some cases.

The quick transient operates on a faster time scale $\bar{t}=t / \epsilon^{*}$. Making this change of variable, we see that to lowest order (2.79) becomes

$$
u_{\bar{t}}=u_{s s}-v u,
$$


and of course (2.78) reduces to (2.74) to lowest order.

If $\rho \ll 1, \kappa(s, 0)>0$, and we assume this equation is still valid, and there exists no source such as occurs for the plate problem described in Section 2.5, then initially we expect $v=\mathrm{O}\left(\frac{1}{\rho}\right) \gg 1$ from (2.74) and hence, from (2.80), $u$ will decay rapidly to 0 . At the same time, $\Gamma(t)$ moves rapidly by a dynamic forced motion by curvature law according to (2.74). In this transient process, there is still a difficult coupling between the dynamics of $u$ and $\Gamma$.

In the case of the plate problem described in Section 2.5 and studied in steady state in Section 3, again when $\rho \ll 1$, we expect that a rapid transient will bring a wide range of initial states to one of the steady solutions of Problem I or II in Section 3.1. The general mechanism of this process, however, is still unknown.

An easier case is when $\rho^{-1}=\mathrm{O}(1)$. Then it is seen from (2.74) that the speed remains $\mathrm{O}(1)$, so that the curve $\Gamma$ moves only a small distance from its initial position $\Gamma(0)$ during the short time evolution governed by the time scale of $\bar{t}$. Therefore the term $\kappa$ in (2.74) may be considered fixed and known, and the value of $v$ obtained therefrom substituted into (2.80) to yield a single equation

$$
u_{\bar{t}}=u_{s s}-\frac{1}{\rho}\left(\kappa+u^{2}\right) u,
$$

a semilinear heat equation to be solved with initial data $u(s, 0)$ given. If $\kappa>0$, the solution can be expected to approach a unique $\bar{t}$-independent solution of (2.81) as $\bar{t} \rightarrow \infty$. In fact, this can be proved for all physically interesting boundary conditions on $\Gamma(t)$, since the nonlinearity in (2.81) is monotone decreasing in $u$.

This $\bar{t}$-limiting state can then be used as initial condition for the evolution (2.74), (2.75) under the slower time scale $t$.

If $\kappa<0$ or $\kappa$ changes sign, then sub- and super-solutions can be used to prove the existence of stable stationary solutions of (2.81), not necessarily unique, which are limits as $\bar{t} \rightarrow \infty$ of wide classes of solutions. Again, the evolution now proceeds under the slower time scale.

\section{Steady motion of a grain boundary with source at one or two ends}

In this section we look for special solutions of (2.74), (2.75) corresponding to experiments involving a metal plate immersed in a solute vapour. Although grooving is an important effect [3], we neglect it in this paper. Accounting for grooving would simply change the boundary conditions (3.4) below.

Problem I below represents a plate of dimensionless thickness $2 H$ with a single grain boundary connecting the two faces. We call this the 'connecting case' (Fig. 2). At the faces there is a vapour with given concentration. Solute is supplied equally at the two plate faces and after diffusing, is deposited in the growing grain. As before, the geometry is 2D. The motion has reached a steady state, so that $\Gamma(t)$ moves without changing shape to the left with speed $\omega$ in a direction along the surface of the plate.

More general solutions of (2.74), (2.75) with appropriate boundary conditions presumably converge to those of the equations below, which are for steady travelling waves.

Symmetry with respect to the mid-line of the plate is assumed. The problem when vapour is present at only one of the two faces can be handled as easily; only obvious changes need be made.

Problem II corresponds to a plate so thick that no steady configuration can exist with the grain boundary passing completely through the plate. The grain boundary does not reach the other side of the plate; it simply trails behind the moving intersection of the boundary with the surface of the plate. We call this the 'trailing case' (Fig. 2). 


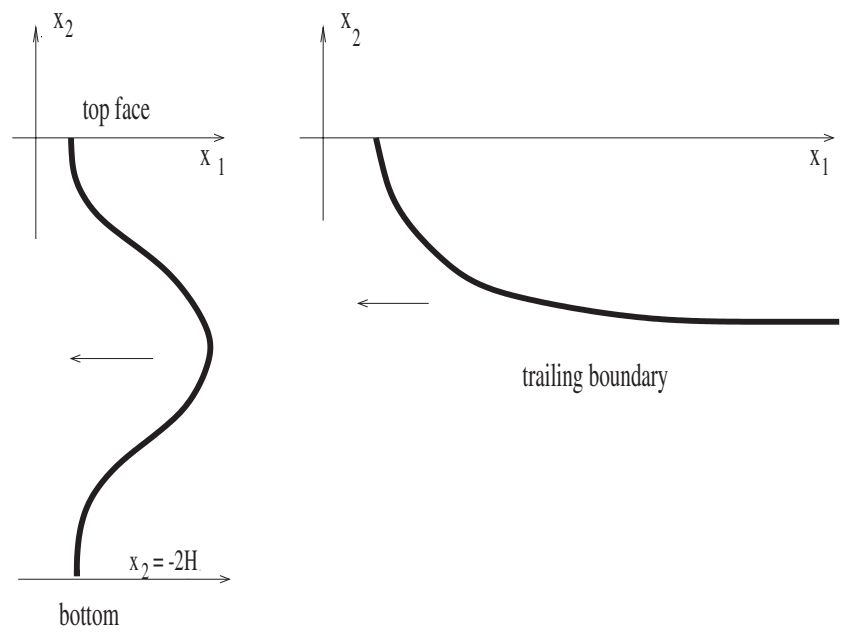

FIG. 2. The connecting and trailing solutions.

The theorems give basic existence of travelling waves for the two problems, together with finiteness of the penetration distance into the metal plate, for our reduced model, in the case of the second problem (see the comment on validity below). We have not as yet been able to prove uniqueness of the solutions of this steady-state model.

For $H$ near a critical value beyond which no solution of Problem I exists, we argue in Section 3.3 that the grain boundary bulges out in the region in the middle of the plate and moves approximately by curvature alone. In that section we approximate the solution in the bulge region and find its thickness width as a function of $\omega$. This gives rise to the conjecture given there about the relation between the critical value of $H$ and the penetration distance $H_{\infty}$ associated with Problem II.

Note. The forcing relation does not have to be $u^{2}$ for most of the following to work. The term $-u^{2}$ in (3.2) can be replaced by $-f(u)$, where $f$ is any differentiable strictly increasing function with $f(0)=0$, and many analogous results continue to hold. For example, in (3.12) below the integrand $u^{2}$ will be replaced by $f(u)$.

\subsection{Problems and results}

In Problem I, $H$ is the half-thickness of the plate. The top face is at $x_{2}=0$ in the $\left(x_{1}, x_{2}\right)$ plane, so the plate occupies the strip $\left\{-2 H \leqslant x_{2} \leqslant 0\right\}$. The migrating grain boundary can be represented in the form $x_{1}=X\left(x_{2}\right)-\omega t$.

We generally formulate the problems in terms of its angle of inclination $\theta=\arctan X^{\prime}\left(x_{2}\right)$, and consider only the top half $\left\{-H \leqslant x_{2} \leqslant 0\right\}$ of the plate; the bottom half is then obtained by symmetry. The steady migration is to the left, and the top half of the grain boundary lags further behind as one goes deeper into the plate, so that $\theta \in\left(-\frac{\pi}{2}, 0\right]$.

Recall that the variable $s$ in (2.75) is dimensionless arclength along the grain boundary. We measure it from the top $\left(x_{2}=s=0\right)$. In Problem I let $S$ be the unknown arclength to the midpoint $\left(x_{2}=-H\right)$, where $\theta(S)=0$. Then we may consider the variables $\theta$ and $u$ each to be a function 
only of $s$. The relation between the function $\theta(s)$ and the Cartesian coordinates of points on $\Gamma$ is given by

$$
x_{2}=-\int_{0}^{s} \cos \theta(\sigma) \mathrm{d} \sigma, \quad x_{1}=-\int_{0}^{s} \sin \theta(\sigma) \mathrm{d} \sigma-\omega t .
$$

We have

$$
\kappa=\theta^{\prime}(s), \quad v=\omega \cos \theta(s) .
$$

Our solutions satisfy $\kappa<0$ near the edge $\{s=0\}$ and $v>0$ everywhere.

In terms of these variables, equations (2.74), (2.75) become (3.2) and (3.3) below.

Problem I-the connecting case. Given numbers $0<\rho \leqslant 1$ and $H>0$, find numbers $\omega>0, S$ and functions $\theta(s), u(s)$ defined on $[0, S]$ such that

$$
\begin{gathered}
\theta^{\prime}(s)=\rho \omega \cos \theta(s)-u^{2}(s), \\
u^{\prime \prime}(s)-\omega \cos \theta(s) u(s)=0, \\
\theta(s) \in(-\pi / 2,0], \quad \theta(0)=0, \quad \theta(S)=0, \\
u(s) \in(0,1], \quad u(0)=1, \quad u^{\prime}(S)=0, \\
\int_{0}^{S} \cos \theta(s) \mathrm{d} s=H .
\end{gathered}
$$

Problem II-the trailing case. Given a number $0 \leqslant \rho \leqslant 1$, find a number $\omega>0$ and functions $\theta(s), u(s)$ defined on $[0, \infty)$ such that (3.2) and (3.3) hold, as well as

$$
\begin{gathered}
\theta(s) \in(-\pi / 2,0], \quad \theta(0)=0, \\
u(s) \in(0,1], \quad u(0)=1 .
\end{gathered}
$$

Comments on the 'trailing solutions'. Because trailing solutions depict a grain boundary which is infinite in extent, travelling for an infinite amount of time, they must only be meant to represent long actual boundaries, and only some finite part of them is physically meaningful. Relevant to this point is the fact that if at least one of the correction terms indicated in (2.79), specifically the term $u_{t}$ on the left, were included in our model, it would result in an extra term ' $-\epsilon^{*} \omega \sin \theta(s) u^{\prime}(s)$ ' on the left of (3.3), which would alter the behaviour of the infinite trailing boundaries at large arclengths $s$ along the boundary. In fact, the boundary would not then have finite penetration depth (distance from the face of the specimen), as indicated in Theorem 2(c) below and alluded to in 2(a). According to a formal analysis, including this term would change the behaviour of the trailing solution from one which has finite overall penetration distance to one whose penetration distance $x_{2}(s)$ grows slowly as we proceed along the grain boundary, namely $x_{2} \simeq \epsilon \ln s$. Moreover, the rates of decay indicated in (3.13) would eventually give place to slower rates: $u(s) \leqslant \mathrm{O}\left((\epsilon / s)^{1 / 2}\right), \theta(s)+\frac{\pi}{2} \leqslant \mathrm{O}(\epsilon / s)$. Since we are always assuming $\epsilon \ll 1$ and $\ln s$ has slow growth, the effect of this perturbation on the penetration depth would probably not be significant when related to any laboratory conditions. For example, the quantity $\epsilon \ln s$ remains less than $5 \epsilon|\ln \epsilon|$ as long as $s \leqslant \epsilon^{-5}$, which is a very long distance. Alternatively, this term would not be significant in these long trailing boundaries, if we 
allowed some diffusion to occur in the grains, with a diffusion coefficient too small to affect DIGM in the experimental time scale.

An important conclusion from the theorems below is that a connecting solution can only be expected if the thickness $H$ of the plate is less than some threshhold thickness. In view of the discussion just given of the effect of neglected terms on very long boundaries, we should modify this conclusion to claim only that it is true under typical laboratory conditions.

\section{THEOREM 1}

(a) Problem II has a solution. It satisfies

$$
u(\infty)=0, \quad \theta(\infty)=-\pi / 2
$$

(b) If $H<\frac{1}{2} \sqrt{\rho}$, there exists a solution of Problem I.

We conjecture that the solution of Problem I is unique, and the same for Problem II. However, it is clearly possible for both a connecting and a trailing solution to exist. For example, if $H$ is larger, but not too much larger, than the penetration distance $H_{\infty}$ given in (3.12) below, then a trailing solution will exist, and no doubt a connecting solution as well. In any case, there are certain properties which all solutions share.

THEOREM 2 There exist functions $H^{*}(\rho)>0$ and $\omega_{*}(\rho)>0$, depending only on $\rho$, such that

(a) The condition

$$
H<H^{*}
$$

is necessary for the existence of a solution of Problem I.

(b) Every solution of Problem I or II satisfies

$$
\omega \geqslant \omega_{*}
$$

(c) For each solution of Problem II, there exists a finite number $H_{\infty}$ satisfying

$$
H_{\infty} \doteq \int_{0}^{\infty} \cos \theta(s) \mathrm{d} s=(\rho \omega)^{-1}\left[\int_{0}^{\infty} u^{2}(s) \mathrm{d} s-\frac{\pi}{2}\right]<\infty
$$

(the second equation holding if $\rho>0$ ). Moreover,

$$
u(s) \leqslant \mathrm{O}\left(s^{-1}\right), \quad \theta(s)+\frac{\pi}{2} \leqslant \mathrm{O}\left(s^{-2}\right)
$$

as $s \rightarrow \infty$.

Part (a) of this theorem means that when the plate's thickness surpasses some critical amount, depending on $\rho$, there is no steady solution representing a grain boundary which extends clear through the plate. Part (c) implies that the steady trailing solution (Problem II) penetrates only a finite distance $H_{\infty}$ into the material. 


\subsection{Proofs}

We shall need the following lemmas.

LEmma 1 Let $(\theta, u, \omega)$ satisfy (3.2), (3.7), and (3.8) for $s \in[0, S]$ for some $0<S \leqslant \infty, 0 \leqslant \rho \leqslant$ 1 , with $\theta(S)=0$ if $S<\infty$ and $S=\infty$ if $\rho=0$. Assume $u(s)>0$ is differentiable, decreasing, and strictly decreasing for small positive $s$. Then

(a) If $\theta^{\prime}\left(s_{1}\right) \geqslant 0$ and $s>s_{1}$, then $\theta^{\prime}(s) \geqslant \theta^{\prime}\left(s_{1}\right)$.

(b) If $S=\infty$, then $\theta$ is a nonincreasing function.

(c) If $S<\infty$, then $\theta^{\prime}(S)>0$.

(d) $\rho \omega<1$.

Lemma 2 Let $(\theta, u, \omega)$ satisfy (3.3) for $s \in[0, S]$ for some $0<S \leqslant \infty$. Assume $\omega \cos \theta(s)>0$, $u(0)=1, u^{\prime}(S)=0$ if $S<\infty$, and $u$ bounded in any case. Then

(a) $u(s)>0$.

(b) $u^{\prime}(s)<0$ when $s<S$.

Proof of Lemma 1 (a) The case $\rho=0$ is trivial, since $\theta^{\prime}=-u^{2}<0$. Assume $\rho>0$. Note that

$$
\theta^{\prime \prime}(s)=-\rho \omega(\sin \theta) \theta^{\prime}-\left(u^{2}\right)^{\prime} .
$$

Let $a>0$, and $e(s)=\exp \left[\rho \omega \int_{a}^{s} \sin \theta(s) \mathrm{d} s\right]$, so that (since $\sin \theta \leqslant 0$ and $\left.\omega>0\right) e$ is nonincreasing in $s$. Multiplying the above equation by $e(s)$, we get

$$
\left(e(s) \theta^{\prime}(s)\right)^{\prime}=-e(s)\left(u^{2}\right)^{\prime} \geqslant 0,
$$

so that for $s>a, \theta^{\prime}(s) \geqslant \theta^{\prime}(a)[e(s)]^{-1} \geqslant \theta^{\prime}(a)$ if $\theta^{\prime}(a) \geqslant 0$.

(b) and (c) follow from (a).

(d) Again the case $\rho=0$ is trivial, so assume $\rho>0$. From (3.2), (3.7), (3.8) we have $\theta^{\prime}(0)=$ $\rho \omega-1$. From (3.7) we must have $\theta^{\prime}(0) \leqslant 0$, i.e. $\rho \omega \leqslant 1$. We can exclude equality here, since in that case $\theta(s)$ would be $>0$ for small $s>0$ due to the fact that $u$ is strictly decreasing.

\section{Proof of Lemma 2}

(a) Since $\omega \cos \theta(s)>0$, the equation (3.3) for $u$ obeys a maximum principle, whether its domain $[0, S]$ be finite or infinite. The function $u \equiv 0$ is a subsolution, so if $u$ is bounded, $u>0$.

(b) From part (a) and (3.3), we have $u^{\prime \prime}>0$. If $u^{\prime}(s) \geqslant 0$ with $s<S$, $u$ could not be bounded if $S=\infty$, nor satisfy $u^{\prime}(S)=0$ if $S<\infty$. So $u^{\prime}(s)<0$.

Lemma 3 Let $S \in(0, \infty), \rho>0$, and let $u(s)>0$ be a continuous decreasing function defined on $[0, S]$, satisfying $u(0)=1, u^{\prime}(s)<0$ for $0<s<s_{1}$ for some $s_{1} \in(0, S)$.

(a) There exists a unique solution $(\theta, \omega)$ of (3.2), (3.4). It depends continuously on $S$.

(b) Both $\theta(s)$ and $\omega$ are strictly decreasing functions of $S$ for $s>0$.

(c) If $u \geqslant \mathrm{e}^{-s / \sqrt{\rho}}$, then

$$
\rho \omega \geqslant \frac{\sqrt{\rho}}{2 S}\left(1-\mathrm{e}^{-2 S / \sqrt{\rho}}\right) \equiv \omega_{0}>0 .
$$


Proof. (a) Consider the initial-value problem for (3.2) with $\theta(S)=0$ and $\omega$ in the interval $u^{2}(S)<$ $\rho \omega \leqslant 1$. Since $\theta^{\prime}(S)=\rho \omega-u^{2}(S)>0$, the solution $\theta(s)$ is near and less than 0 for $s$ near and less than $S$. Also $\theta(s)>-\frac{\pi}{2}$ for $0 \leqslant s \leqslant S$, since $\theta^{\prime}(s)$ would have to be non-negative at the last place where $\theta=-\frac{\pi}{2}$, contradicting (3.2).

Let $M$ be the set of values of $\omega$ such that $u^{2}(S)<\rho \omega \leqslant 1$ and the solution of the initial value problem satisfies

$$
\theta(0) \leqslant 0 .
$$

First, we show that $\frac{1}{\rho} \in M$. Setting $\omega=\frac{1}{\rho}$ implies that $-\frac{\pi}{2}<\theta(s)<0$ for $s \in(0, S)$; in fact, if $\theta\left(s^{*}\right)=0$ for $s^{*}$ in that interval (let it be the last one), then $\theta^{\prime}\left(s^{*}\right) \leqslant 0$, which contradicts (3.2) and the decreasing nature of $u$.

Now let $\omega^{*}=\inf M$. Integrating (3.2) with $\omega=\omega^{*}$ after dividing by $\cos \theta$, we find

$$
S \rho \omega^{*}=\int_{\theta(0)}^{0} \sec \theta \mathrm{d} \theta+\int_{0}^{S} u^{2}(s) \sec \theta(s) \mathrm{d} s>\int_{0}^{S} u^{2}(s) \mathrm{d} s>S u^{2}(S),
$$

so that $\omega^{*}$ lies in the interior of the allowed $\omega$-interval. But the continuity of the solution with respect to $\omega$ implies that if $\theta(0)<0, \omega^{*}$ could not be the infimum. Therefore, the solution $\theta(s)$ associated with $\omega^{*}$ must satisfy $\theta(0)=0$.

This provides existence. The uniqueness follows from the monotonicity in $\omega$ to be proved next (which shows that two solutions must have the same $\omega$ ), together with the uniqueness of solutions of the initial-value problem. The continuity of the solution of the above initial-value problem with respect to $S$, together with the monotonicity, imply that the solution $(\theta, \omega)$ depends continuously on $S$.

(b) For any number $\omega \in\left(0, \frac{1}{\rho}\right)$, let $\theta_{\omega}(s)$ be the solution of (3.2) with $\theta_{\omega}(0)=0$. Let $\phi(s)=$ $\frac{\partial}{\partial \omega} \theta_{\omega}(s)$, so that as long as $\cos \theta_{\omega} \in\left(-\frac{\pi}{2}, 0\right]$,

$$
\phi^{\prime}+\rho \omega \sin \left(\theta_{\omega}(s)\right) \phi=\rho \cos \theta_{\omega}(s)>0, \quad s \in(0, S] .
$$

This, together with $\phi(0)=0$, implies $\phi(s)>0$. We conclude that $\theta_{\omega}$ is strictly increasing and continuous in $\omega$ as long as $\cos \theta_{\omega} \in\left(-\frac{\pi}{2}, 0\right]$.

Now consider a number $S_{0}>0$ and the solution $\left(\theta_{0}, \omega_{0}\right)$ considered in the proof of (a) corresponding to this value of $S$. Let $S_{1}>S_{0}$ and let $\left(\theta_{1}, \omega_{1}\right)$ be its corresponding solution. Then $\omega_{1}<\omega_{0}$ because if $\omega_{1} \geqslant \omega_{0}$, the monotonicity with respect to $\omega$ shown above would imply $\theta_{1}\left(S_{0}\right) \geqslant \theta_{0}\left(S_{0}\right)=0$, which is a contradiction. Therefore, $\omega$ is a strictly decreasing function of $S$.

The other part of (b) follows from this since $\theta_{\omega}$ is increasing in $\omega$.

(c) An upper bound for the solution of (3.2), (3.4) is provided as follows. $\theta^{\prime} \leqslant \rho \omega-u^{2}$, hence $\theta(s) \leqslant \rho \omega s-\int_{0}^{s} u^{2}(t) \mathrm{d} t$. Therefore, $\theta(s)<0$ for $s \in(0, \hat{S})$ if

$$
\rho \omega<\inf _{s \in(0, \hat{S}]}\left[s^{-1} \int_{0}^{s} u^{2}(t) \mathrm{d} t\right] \doteq \tilde{\omega}[u, \hat{S}]>0 .
$$

Thus when $\rho \omega<\tilde{\omega}[u, \hat{S}], S>\hat{S}$.

Set $\underline{u}=\mathrm{e}^{-s / \sqrt{\rho}}$ and $\omega_{0}(\rho, S)=\tilde{\omega}[\underline{u}, S]>0$. If $\rho \omega<\omega_{0}$, then also $\rho \omega<\tilde{\omega}(u, S)$ if $u \geqslant \underline{u}$. This would yield the contradiction $S>S$. For our solution we must therefore have $\rho \omega \geqslant \omega_{0}$. A calculation yields that this number $\omega_{0}$ is given by (3.15). 
LEMmA 4 Given $S \in(0, \infty)$ and a continuous function $\omega \cos \theta(s)$ such that $0 \leqslant \omega \cos \theta(s) \leqslant \frac{1}{\rho}$ for $0 \leqslant s \leqslant S$, there exists a unique solution of (3.3), (3.5), on [0,S]. Moreover,

$$
\mathrm{e}^{-s / \sqrt{\rho}} \leqslant u(s) \leqslant 1 .
$$

Proof. The functions $\bar{u} \equiv 1$ and $\underline{u} \equiv \mathrm{e}^{-s / \sqrt{\rho}}$ are super- and sub-solutions of (3.3), (3.5). To check the latter, note that

$$
\underline{u}^{\prime \prime}-\omega \cos \theta \underline{u}=\left(\frac{1}{\rho}-\omega \cos \theta\right) \underline{u} \geqslant 0, \quad \underline{u}(0)=1, \quad \underline{u}^{\prime}(S)<0 .
$$

Thus there exists an exact solution between them. It is unique, by the maximum principle.

Proof of Theorem 1(a) in the case $\rho>0$. First, for any given finite $S>0$, we prove the existence of a solution of (3.2)-(3.5). (This is Problem I with the last condition (3.6) deleted and $S$ prescribed.)

Given $S>0$, let $K_{S}=\{(\theta, \omega): \theta$ a continuous function on $[0, S],-\pi / 2 \leqslant \theta(s) \leqslant 0$, $\theta(0)=\theta(S)=0$, and $\left.\omega_{0}(\rho, S) \leqslant \rho \omega \leqslant 1\right\}$, where $\omega_{0}$ is given by (3.15). We think of $K_{S}$ as a closed convex set in the Banach space of pairs $(\theta, \omega)$, where $\theta$ is given the $L_{\infty}$ norm.

We define a mapping $F$ from $K_{S}$ into itself. This is done in two steps. We start with a given $(\tilde{\theta}, \tilde{\omega}) \in K_{S}$.

(i) Solve the following for a function $u(s)>0$ :

$$
\begin{gathered}
u^{\prime \prime}(s)-\tilde{\omega} \cos \tilde{\theta}(s) u(s)=0, \\
u(0)=1 ; \quad u^{\prime}(S)=0 .
\end{gathered}
$$

The existence of a unique solution follows from Lemma 4. Moreover, $u \in C^{2}([0, S])$ with corresponding norm bounded independently of everything. From Lemma $2(\mathrm{~b}), u^{\prime}(s)<0$ when $s<S$.

(ii) Solve (3.2), (3.4) with the function $u$ already found in (i) inserted into (3.2), for the pair $(\theta, \omega)$.

Again, the existence of a unique solution follows from Lemma 3. Moreover, because of the regularity of $u$ mentioned above, $\theta \in C^{3}([0, S])$, with corresponding norm bounded in terms of only $\rho$. From Lemma 4, we have bounds on $u$ which imply, by Lemmas 3(c) and 1(d), that $\omega_{0}(\rho, S) \leqslant \rho \omega<1$.

This defines the map $(\theta, \omega)=F(\tilde{\theta}, \tilde{\omega})$ from $K_{S}$ into itself. Also $F$ is compact, due to the estimates on the derivatives of $u$ and $\theta$ indicated above. The existence of a fixed point follows from the Schauder theorem. This fixed point, together with the associated function $u$, will satisfy (3.2)(3.5).

Next we obtain a solution of Problem II by passing to the limit $S \rightarrow \infty$. Again, the above estimates on derivatives of $u$ and $\theta$ show that they form an equicontinuous family, depending on the parameter $S$. On each $s$-interval $I_{k}=[0, k]$ ( $k$ an integer), there is therefore a subsequence $S_{n_{k}} \rightarrow \infty$ along which the corresponding solutions $\left(\theta_{n_{k}}, u_{n_{k}}, \omega_{n_{k}}\right)$ converge uniformly to a limit. A diagonal subsequence converges everywhere to a limit $(\theta, u, \omega)$ which satisfies (3.2), (3.3), (3.7) and (3.8).

We finally prove (3.9). By the monotonicity of $\theta$ and $u$ (Lemmas 1(b) and 2(b)) $\theta(\infty)$ and $u(\infty)$ exist. And by (3.2), $\rho \omega \cos \theta(\infty)=u^{2}(\infty)$. If the two sides of this equation did not vanish, then 
the coefficient $\omega \cos \theta(s)$ of $u$ in (3.3) would be positive and bounded away from zero. Therefore, by comparison with a solution of the same equation with that coefficient replaced by a positive constant, we would have that $u$ decays to 0 as $s \rightarrow \infty$, a contradiction.

Therefore $u(\infty)=0=\omega \cos \theta(\infty)$. Thus either $\theta(\infty)=-\frac{\pi}{2}$ or $\omega=0$. If $\omega=0$, (3.3), (3.5), would imply $u \equiv 1$, which is impossible since $u(\infty)=0$. Therefore $\omega \neq 0$ and $\theta(\infty)=-\frac{\pi}{2}$. This completes the proof.

Proof of Theorem 1(a) in the case $\rho=0$. We start with an auxiliary problem: given $S>\pi$ and $\delta>0$, solve

$$
\begin{gathered}
\theta^{\prime}(s)=-u^{2}(s), \quad \theta(0)=0, \quad \theta(S)=-\frac{\pi}{2}+\delta, \\
u^{\prime \prime}(s)=\omega \cos \theta(s) u(s), \quad u(0)=1, \quad u^{\prime}(S)=0 .
\end{gathered}
$$

Let $g(k)=\frac{1-\mathrm{e}^{-k}}{k}$; it is a decreasing function of $k>0$ satisfying $g(0)=1, g(\infty)=0$. Let $\omega_{1}<\omega_{2}$ satisfy

$$
\operatorname{Sg}\left(2 \sqrt{\omega_{2} \alpha} S\right)<\frac{\pi}{2}-\delta<S g\left(2 \sqrt{\omega_{1}} S\right),
$$

where $\alpha=\cos \left(-\frac{\pi}{2}+\delta\right)>0$. This is possible by the properties of $g$ and the fact that $S>\pi$.

Let $K$ be the set of all continuous functions $\theta(s), 0 \leqslant s \leqslant S$ satisfying $\theta(0)=0, \theta(S)=$ $-\frac{\pi}{2}+\delta, \theta(s) \in\left[-\frac{\pi}{2}+\delta, 0\right]$. We supply $\theta$ with the uniform norm and construct a compact mapping from $K$ into itself.

Given $\tilde{\theta} \in K$,

(i) solve for $u$ and $\omega$ :

$$
u^{\prime \prime}=\omega \cos \tilde{\theta} u, \quad u(0)=1, \quad u^{\prime}(S)=0, \quad \int_{0}^{S} u^{2} \mathrm{~d} s=\frac{\pi}{2}-\delta
$$

We show that a unique solution exists. If $\omega>\omega_{2}$, then $\omega \cos \tilde{\theta}>\omega_{2} \alpha$, and by an estimate like that in the proof of Lemma $4, u<\mathrm{e}^{-\sqrt{\omega_{2} \alpha} s}$. Hence

$$
\int_{0}^{S} u^{2} \mathrm{~d} s<S g\left(2 \sqrt{\omega_{2} \alpha} S\right)<\frac{\pi}{2}-\delta .
$$

On the other hand, if $\omega<\omega_{1}$, by the analogous argument,

$$
\int_{0}^{S} u^{2} \mathrm{~d} s>S g\left(2 \sqrt{\omega_{1}} S\right)>\frac{\pi}{2}-\delta
$$

Since the solution $u$ depends continuously and monotonically on $\omega$, there is a unique value of $\omega \in\left(\omega_{1}, \omega_{2}\right)$ for which the integral condition is satisfied.

(ii) With this value of $u$, set $\theta(s)=-\int_{0}^{s} u^{2}(t) \mathrm{d} t$. This function is in $K$.

This gives a mapping of $K$ into itself; it is compact because a priori estimates on derivatives of the image follow from the differential equations. It therefore has a fixed point, which will generate, for given $S, \delta$, a solution of the problem (3.19), (3.20).

We now let $S \rightarrow \infty$ and $\delta \downarrow 0$. A standard equicontinuity argument shows the existence of a subsequence approaching a solution of the parts of (3.19), (3.20) not involving $S$. Moreover, the 
limit functions will have ranges in the intervals $u(s) \in[0,1], \theta(s) \in\left(-\frac{\pi}{2}, 0\right)$. By the dominated convergence theorem, we have

$$
\int_{0}^{\infty} u^{2}(s) \mathrm{d} s=\frac{\pi}{2},
$$

and since $u$ is monotone decreasing, it satisfies $u(\infty)=0$. Similarly, $\theta(\infty)=-\frac{\pi}{2}$. This finishes the proof of Theorem 1(a) in the case $\rho=0$.

Proof of Theorem (1b). We shall use the notation $s=\xi S, u(\xi S)=v_{S}(\xi), \theta(\xi S)=\phi_{S}(\xi)$. We work with the functions $\phi$ and $v$ on the interval $0 \leqslant \xi \leqslant 1$. Let $M$ be the following closed convex set in the Banach space of triples $(\phi, \omega, S)$, where $\phi \in C^{0}([0,1])$ with the uniform norm, and $\omega$ and $S$ are real numbers:

$$
M=\left\{(\phi, \omega, S): \phi(0)=\phi(1)=0, \quad-\frac{\pi}{2} \leqslant \phi(s) \leqslant 0, \omega_{0} \leqslant \rho \omega \leqslant 1,0 \leqslant S \leqslant T\right\},
$$

where $\omega_{0}>0$ will be chosen later and $T$ is chosen so large that

$$
H<\frac{1}{2} \sqrt{\rho}\left(1-\mathrm{e}^{-2 T / \sqrt{\rho}}\right) .
$$

This is possible by the assumption on $H$.

We now define a compact mapping from $M$ into itself, in three steps. Let $(\tilde{\phi}, \tilde{\omega}, \tilde{S})$ be an arbitrary element of $M$.

(i) Solve the following for a function $v(\xi), \xi \in[0,1]$ :

$$
\ddot{v}-\tilde{S}^{2} \tilde{\omega}(\cos \tilde{\phi}) v=0, \quad v(0)=0, \quad \dot{v}(1)=0 .
$$

where dots mean $\frac{d}{d \xi}$.

The solution exists by a slightly altered Lemma 4 , which provides the bounds

$$
\mathrm{e}^{-\tilde{S} \xi / \sqrt{\rho}}<v(\xi) \leqslant 1 .
$$

(ii) Let

$$
u(s)= \begin{cases}v\left(\frac{s}{\tilde{S}}\right), & s \leqslant \tilde{S} \\ v(1), & s \geqslant \tilde{S}\end{cases}
$$

By (3.28), it satisfies

$$
u(s) \geqslant \mathrm{e}^{-s / \sqrt{\rho}} .
$$

For each $S \leqslant T$, solve for $\theta=\theta_{S}(s), \omega=\omega_{S}$ :

$$
\theta^{\prime}=\rho \omega \cos \theta-u^{2}, \quad \theta(0)=\theta(S)=0, \quad \theta \in\left(-\frac{\pi}{2}, 0\right] .
$$

Lemma 3(a) provides a unique solution, depending continuously on $S$. Moreover, by Lemma $3(\mathrm{~b}), \omega_{S}$ is a decreasing function of $S$ and satisfies (3.15). The argument leading to (3.15) now yields the lower bound

$$
\rho \omega_{S} \geqslant \frac{\sqrt{\rho}\left(1-\mathrm{e}^{-2 S / \sqrt{\rho}}\right)}{2 S}=g(2 S / \sqrt{\rho}) \geqslant g(2 T / \sqrt{\rho}) \equiv \omega_{0},
$$


where $g$ is the function in (3.21). This is the value of $\omega_{0}$ we use in the definition of $M$.

Now define $h(S)=\frac{1}{\rho \omega_{S}} \int_{0}^{S} u^{2}(s) \mathrm{d} s$, a continuous function of $S$ with $h(0)=0$. We wish to choose $S<T$ so that

$$
H=h(S)
$$

By the decreasing nature of $\omega_{S}$, we see that $h(S)$ is an increasing function of $S$. Therefore, there will be a unique solution of (3.33) with the required bound provided that $h(T)>H$. By the lower bound (3.30), the estimate $\omega_{S}<\frac{1}{\rho}$ from Lemma 1(d) and the definition (3.26), we determine that

$$
\begin{aligned}
h(T) & \geqslant \int_{0}^{T} \mathrm{e}^{-2 s / \sqrt{\rho}} \mathrm{d} s \\
& =\frac{1}{2} \sqrt{\rho}\left(1-\mathrm{e}^{-2 T / \sqrt{\rho}}\right)>H .
\end{aligned}
$$

The next step is therefore possible:

(iii) Select $S<T$ such that $h(S)=H$.

With this choice of $S$, we set $\phi(\xi)=\theta_{S}(S \xi), \omega=\omega_{S}$, and the definition of our map from $M$ into itself is complete. The mapping is compact; in fact, by (3.27) and (3.29), $\ddot{u}$ has a uniform bound, and hence from (3.31), $\theta_{S}$ and therefore the new function $\phi$ has derivative bounded independently of the initial function $\tilde{\phi}$.

The map therefore has a fixed point $(\phi, \omega, S)$. The corresponding functions $\theta(s)=$ $\phi(s / S), u(s)=v(s / S)$ provide the solution of Problem I. In fact, equations (3.2)-(3.5) are immediate, and we need only check (3.6). But that also follows from (3.33) and integrating (3.2) from 0 to $S$.

We now turn to the proof of Theorem 2, for which we need the following two lemmas.

LEMma 5 Let $S>0$ and let the functions $u, \psi$ satisfy

$$
\begin{gathered}
u^{\prime \prime}-\psi u=0, \quad 0<s<S, \\
u(0)=1, \quad u^{\prime}(S)=0, \quad \psi>0, \\
a u^{2}+b u u^{\prime}<\psi
\end{gathered}
$$

for some positive numbers $a$ and $b$.

Then there exists a number $m$, depending on $a$ and $b$ but not on $S$, such that

$$
0<u(s)<\frac{m}{s+1}
$$

The same conclusion holds if $S=\infty$ and the boundary condition at $S$ is replaced by $u(\infty)=0$.

Proof. Note that $u>0, u^{\prime}<0$ by Lemma 2. Consider the nonlinear differential operator

$$
N_{S}[v] \doteq v^{\prime \prime}-\left(a v^{2}+b v v^{\prime}\right) v, \quad 0<s<S,
$$

with associated boundary conditions $v(0)=1, \quad v^{\prime}(S)=0$. Now $u$ is a subfunction for $N_{S}$, because (3.36) shows that $N_{S}[u]>0$. 
We also construct a superfunction in the form

$$
\bar{v}=\frac{A}{s+B}+\epsilon s
$$

for $A$ and $B$ to be specified below, and $\epsilon=\frac{2 A}{(S+B)^{2}}$ designed so that $\bar{v}^{\prime}(S)>0$. We write

$$
N_{S}[\bar{v}]=\bar{v}^{\prime \prime}+\bar{v}^{2}\left(-a \bar{v}+b\left(-\bar{v}^{\prime}\right)\right)
$$

and use the facts that $-\bar{v}^{\prime}<\frac{A}{(s+B)^{2}}$ and $\bar{v}>\frac{A}{s+B}$ to estimate

$$
-a \bar{v}+b\left(-\bar{v}^{\prime}\right)<\frac{A}{s+B}\left[-a+\frac{b}{s+B}\right] .
$$

We now choose $B=\frac{2 b}{a}$ so that the bracket on the right $\leqslant-\frac{a}{2}<0$, and hence

$$
N_{S} \bar{v}<\bar{v}^{\prime \prime}-\bar{v}^{2} \frac{a A}{2(s+B)}<\frac{A}{(s+B)^{3}}\left[2-\frac{A^{2} a}{2}\right] .
$$

We finally choose $A$ so large that $N_{S} \bar{v}<0$, and also $\bar{v}(0)>1$. Both $A$ and $B$ are independent of $S$.

We now have a superfunction $\bar{v}$ and a subfunction $u$. We must show specifically that this implies $u \leqslant \bar{v}$, because $N_{S}$ does not enjoy a general comparison principle. If it were not true, then $w=\bar{v}-u$ would have a negative minimum at some point $s_{0}$, which cannot be 0 or $S$, by our choice of boundary conditions on $\bar{v}$. Therefore, at $s_{0} \in(0, S)$ we have $w^{\prime}=0, w^{\prime \prime} \geqslant 0, \bar{v}<u$. Now

$$
\begin{gathered}
0>N_{S}[\bar{v}]-N_{S}[u]=w^{\prime \prime}-a\left(\bar{v}^{3}-u^{3}\right)-b\left(\bar{v}^{2}-u^{2}\right) \bar{v}^{\prime} \\
\geqslant(u-\bar{v})\left[a\left(\bar{v}^{2}+u^{2}\right)+b \bar{v}^{\prime}(\bar{v}+u)\right] \\
\geqslant(u-\bar{v})(u+\bar{v})\left(a \bar{v}+b \bar{v}^{\prime}\right) .
\end{gathered}
$$

If $a \bar{v}+b \bar{v}^{\prime}>0$ at $s_{0}$, then we obtain a contradiction, proving that $u \leqslant \bar{v}$. but

$$
a \bar{v}+b \bar{v}^{\prime}=\frac{A}{s+B}\left(a-\frac{b}{s+B}\right)+\epsilon(s+1)>0
$$

by our previous choice of $B$. Hence

$$
u(s) \leqslant \bar{v}(s)=\frac{A}{s+B}+\epsilon s
$$

for $0 \leqslant s \leqslant S$. Since $\epsilon s \leqslant \frac{2 A s}{(S+B)^{2}}<\frac{2 A}{s+B}$, we obtain the desired conclusion (3.37) if $S<\infty$.

The case $S=\infty$ may be handled as follows. In the above proof, replace $S$ by any finite positive number $S^{*}$, and use, as subfunction, the restriction of $u$ to $\left[0, S^{*}\right]$. The superfunction $\bar{v}$ remains the same. We thus obtain (3.37) for $s \in\left[0, S^{*}\right]$ with $m$ independent of $S^{*}$. It therefore holds for all $s$.

LEMMA 6 For some $S>0$ and $\omega_{*}>0$, let $\theta$ and $u$ satisfy (3.2), (3.4), $u>0, u^{\prime}<0, u^{\prime \prime}>$ $0, \omega \geqslant \omega_{*}$. Then there exist positive $a$ and $b$, depending only on $\omega_{*}$ and $\rho$, such that for $s \in[0, S]$,

$$
a u^{2}+b u u^{\prime}<\omega \cos \theta .
$$

If $\theta$ and $u$ satisfy (3.2), (3.3), (3.7), (3.8), then (3.39) holds for all $s$. 
Proof. We define

$$
p(s) \doteq \frac{\rho \omega \cos \theta(s)}{\theta(s)+\pi / 2} .
$$

Clearly

$$
\frac{2 \rho \omega}{\pi}<p(s)<\rho \omega
$$

for all $s$. Equation (3.2) can be written

$$
\theta^{\prime}-p(s)\left(\theta+\frac{\pi}{2}\right)=-u^{2} .
$$

Multiplying (3.41) by the integrating factor $\mathrm{e}^{-\int p \mathrm{~d} s}$, we learn that $\int_{0}^{S} \mathrm{e}^{-\int_{0}^{s} p(t) \mathrm{d} t} u^{2}(s) \mathrm{d} s=$ $\frac{\pi}{2}\left(1-\mathrm{e}^{-\int_{0}^{S} p(s) \mathrm{d} s}\right)$ and

$$
\begin{aligned}
\theta(s)+\frac{\pi}{2} & =\int_{s}^{S} \exp \left[\int_{t}^{s} p(\tau) \mathrm{d} \tau\right] u^{2}(t) \mathrm{d} t+\frac{\pi}{2} \mathrm{e}^{-\int_{s}^{S} p(t) \mathrm{d} t} \\
& =\int_{s}^{S}\left(u^{2}(t)-u^{2}(s)\right) \exp \left[\int_{t}^{s} p(\tau) \mathrm{d} \tau\right] \mathrm{d} t+u^{2}(s) q(s)+\frac{\pi}{2} \mathrm{e}^{-\int_{s}^{S} p(t) \mathrm{d} t},
\end{aligned}
$$

where by (3.40) $q(s)=\int_{s}^{S} \exp \left[-\int_{s}^{t} p(\tau) \mathrm{d} \tau\right] \mathrm{d} t>\frac{1}{\rho \omega}\left(1-\mathrm{e}^{-\rho \omega(S-s)}\right)$. Since $u \leqslant 1$, the last term in $(3.43) \geqslant u^{2}(s) \frac{\pi}{2} \mathrm{e}^{-\rho \omega(S-s)}$. Hence, since $\rho \omega<1$ from Lemma $1(\mathrm{~d})$, the sum of the last two terms $\geqslant u^{2}(s)$.

Using the convexity of $u^{2}$, we may replace the factor $u^{2}(t)-u^{2}(s)$ in the integrand of (3.43) by $(t-s)\left(u^{2}\right)^{\prime}(s)$, thereby converting (3.43) into an inequality with ' $\geqslant$ '. We note that $\left(u^{2}\right)^{\prime}(s)=$ $2 u(s) u^{\prime}(s)<0$. Since (from (3.40))

$$
2 \int_{s}^{\infty}(t-s) \exp \left[-\int_{s}^{t} p(\tau) \mathrm{d} \tau\right] \mathrm{d} t \leqslant \frac{\pi^{2}}{2 \rho^{2} \omega^{2}},
$$

we get from (3.43)

$$
\theta(s)+\frac{\pi}{2} \geqslant u^{2}(s)+\frac{\pi^{2}}{2 \rho^{2} \omega^{2}} u(s) u^{\prime}(s)
$$

Therefore

$$
\omega \cos \theta \geqslant \frac{2 \omega}{\pi}(\theta+\pi / 2) \geqslant \frac{2 \omega_{*}}{\pi} u^{2}+\frac{\pi}{\rho^{2} \omega_{*}} u u^{\prime} .
$$

Hence the desired conclusion (3.39).

This proves the lemma in the case $S<\infty$. The other case is when (3.2), (3.3) hold for all $s>0$. The proof in this case is obtained by simply replacing $S$ by $\infty$ in the above proof.

Proof of Theorem 2(b). Let $\omega_{*}=\inf \hat{C}$, where $\hat{C}$ is the set of all possible velocities $\omega$ for solutions of Problem I or II, for fixed $\rho$. Since $\rho \omega \in(0,1)$, we have $\omega_{*} \geqslant 0$. We have only to show strict 
inequality here. There exists a sequence $\left(\theta_{k}, \omega_{k}, u_{k}\right)$ of solutions with $\omega_{k} \downarrow \omega_{*}$. Let $S_{k} \leqslant \infty$ be the associated values of $S$. We may also require that $S_{k}$ converge to some limit $S_{\infty} \leqslant \infty$. Now $S_{\infty}>0$ (it may be infinite) because an easy argument shows that $\omega$ is near $\frac{1}{\rho}$ when $S$ is small.

The common bounds for all the functions $\theta_{k}$ and $u_{k}$ and equations (3.2) and (3.3) imply their second derivatives are uniformly bounded and equicontinuous. A standard compactness argument shows that there is a subsequence converging to a solution of Problem I or II on $\left(0, S_{\infty}\right)$, where $0<S_{\infty} \leqslant \infty$, with velocity $\omega=\omega_{*}$. If the latter were 0 , then $u \equiv 1$ by (3.3), and in view of (3.2), our limit solution of (3.2) could not satisfy (3.4) or (3.7).

Proof of Theorem 2(c). Lemma 6 guarantees that the solution satisfies the hypotheses of Lemma 5, where we set $\psi=\omega \cos \theta$. The upper bound (3.37) so obtained on $u$ shows the first part of (3.13) and that $\int_{0}^{\infty} u^{2}(s) \mathrm{d} s<\infty$. Integrating (3.2), we find that for all $s$,

$$
\theta(s)=\rho \omega \int_{0}^{s} \cos \theta(t) \mathrm{d} t-\int_{0}^{s} u^{2}(t) \mathrm{d} t .
$$

Passing to the limit as $s \rightarrow \infty$, we obtain (3.12). The last part of (3.13) follows from $\theta^{\prime}=\rho \omega \cos \theta-$ $u^{2}<0$.

Proof of Theorem 2(a). The conclusion (3.37) of Lemma 5 implies that $\int_{0}^{S} u^{2}(s) \mathrm{d} s$ is bounded independently of $S$. The upper bound $H^{*}$ on $H$ follows from this, the fact that $H=\frac{2}{\rho \omega} \int_{0}^{S} u^{2}(s) \mathrm{d} s$, and the lower bound $\omega \geqslant \omega_{*}$ in (3.11).

\subsection{Critical value of $H$}

For fixed $\rho>0$, we expect that there is a value $H_{c}(\rho)$ of $H$ such that a solution of Problem I exists if and only if $H<H_{c}$. Theorem 1(b) would then provide a lower bound: $H_{c} \geqslant \frac{1}{2} \sqrt{\rho}$, although it will be shown below that there may be reason to believe it is much larger when $\rho$ is small. It is also reasonable that for $H$ slightly less than $H_{c}$, the upper part of the grain boundary can be approximated by the solution of Problem II, which extends to infinity in the direction of the positive $x_{1}$-axis. The mirror image of such a configuration would be attached to the bottom of the plate. What would result is a long bulge in the middle of the plate, where the two approximate solutions of Problem II are connected one with the other. In the region of this bulge, points on the grain boundary will correspond to arclengths which are very large, so that solute material would have to diffuse far from the sources on the plate faces. It will then be reasonable to neglect the solution concentration (and therefore the solute motive force) in that region. Setting $u=0$ in (3.2), approximating the bulge by one of infinite extent, and resetting the origins of both $s$ and $x_{2}$ to the midpoint of the bulge, we obtain the following equation, with boundary conditions, for $\theta(s)$ :

$$
\begin{gathered}
\theta^{\prime}(s)=\rho \omega \cos \theta(s), \\
\theta(0)=0, \quad \theta( \pm \infty)= \pm \frac{\pi}{2} .
\end{gathered}
$$

This can be solved immediately in the form

$$
\theta(s)=-\rho \omega x_{2}(s),
$$

since (3.1) $x_{2}=-\int_{0}^{s} \cos \theta\left(s^{\prime}\right) \mathrm{d} s^{\prime}$. Thus $x_{2}$ passes from $\frac{\pi}{2 \rho \omega}$ to $-\frac{\pi}{2 \rho \omega}$ as $s$ goes from $-\infty$ to $\infty$. Note that this approximation is only for the part of the grain boundary far downstream. In it, the speed $\omega$ is assumed to be known. It will be (approximately) the value associated with Problem II. 
To summarize, the configuration will consist of two solutions of Problem II, one the mirror image of the other and each penetrating a distance $H_{\infty}$, together with a bulge of thickness $\frac{\pi}{\rho \omega}$ connecting them. Recalling that $H$ is defined as the half-thickness of the plate, we obtain the following conjecture about the value of $H_{c}$.

CONJECTURe There exists a (connecting) solution of Problem I if and only if $H<H_{\infty}+\frac{\pi}{2 \rho \omega}$, where $\omega$ and $H_{\infty}$ are the speed and penetration depth corresponding to Problem II (the trailing solution).

\subsection{Slow and fast motion when $\rho \ll 1$}

When $\rho$ is small, Theorem 1(b) gives the existence of a spanning configuration moving with high speed, when the thickness $H$ is small, namely less than $\frac{1}{2} \sqrt{\rho}$. The reason the speed is large can be seen from the proof of that part of the theorem. In fact (3.25) gives the lower bound $\omega \geqslant \frac{\omega_{0}}{\rho}$, and (3.32) yields $\omega_{0}=g(2 T / \sqrt{\rho}) \geqslant C \sqrt{\rho}$ for some $C$, small enough $\rho$, and fixed $T$ (if $\rho$ is small enough, we may select a fixed $T$ to satisfy (3.26)). Therefore $\omega \geqslant \frac{C}{\sqrt{\rho}} \gg 1$.

These highly mobile boundaries have the property that the curvature term on the left of (3.2) is negligible compared to the others, so that $\omega \approx \frac{\overline{u^{2}}}{\rho}$, bar denoting some average. Since $H$ is small, $u$ is not too small, due to its sources at the faces of the thin plate. Therefore $\omega$ is large.

On the other hand, the bulging solutions whose existence we surmised in the previous section have moderate speed. Their speed, in fact, is determined approximately by that for the trailing solution of Problem II with $\rho=0$. In the trailing parts of the configuration, the left side of (3.2) balances the last term on the right, the first term on the right being negligible.

In the bulging part, however, the concentration is negligible and the curvature term on the left of (3.2) balances the first term on the right, which (since $\omega$ is now a known $\mathrm{O}(1)$ quantity) is $\mathrm{O}(\rho)$. The bulge therefore has small curvature and thickness of the order $\frac{1}{\rho}$.

Estimates for typical values of our parameters are given in Section 4. It is noted there that in typical cases, $\rho$ is so small that the width of the bulging configuration would be too large to be observable in practice.

We therefore have two kinds of solutions when $\rho \ll 1$ : rapid ones when $H$ is sufficiently small, and slower ones when $H$ takes large values in a certain range. The existence of solutions for values of $H$ intermediate between these ranges is an open question.

\section{Parameter estimation and discussion}

We began the modelling process by a set of phase field equations (2.1)-(2.4) in which the following dimensional parameters appeared:

$\delta=$ a length characteristic for the gradient contributions to the free energy, and also a measure of the grain boundary width;

$\hat{\epsilon}=$ a nondimensional ratio of elastic energy density, a product of elastic modulus and

Vegard's law coefficient squared, to alloy mixing free energy;

$D_{m}=$ an average diffusivity of solute in the grain boundary;

$c^{\dagger}=$ the maximum value of the concentration; 
$\tau=$ a relaxation time for grain boundary motion;

$\beta=$ a measure of the ratio of interfacial to chemical energy.

In terms of these, our asymptotic analysis proceeded under the basic assumptions (2.13):

$$
\hat{\epsilon} \beta \ll 1, \quad c^{\dagger} \sqrt{\hat{\epsilon} A} \ll 1, \quad \frac{\hat{\epsilon}\left(c^{\dagger}\right)^{2}}{A} \leqslant 1,
$$

which assume the dimensionless form (2.14).

We constructed (formally) solutions which can be approximated by solutions of our freeboundary problem. Those solutions have the following characteristic lengths, which can be read off from assumption I (2.32): the thickness of the grain boundary is $\mathrm{O}(\delta)$, the characteristic radius of curvature of the grain boundary is at least $\frac{\delta}{\hat{\epsilon}\left(c^{\dagger}\right)^{2}} \gg \delta$, and the characteristic length associated with the variation of the concentration $\hat{c}$ along the grain boundary is not less than $\rho^{1 / 2} \delta / \epsilon=\sqrt{\frac{D_{m} \tau}{\hat{\epsilon}\left(c^{\dagger}\right)^{2}}}$. The characteristic velocity of the grain boundary is no larger than $\frac{\hat{\epsilon}\left(c^{\dagger}\right)^{2} \delta}{\tau}$.

One of the basic PDEs, namely (2.3) and its various subsequent versions, has a degenerate diffusivity $D$. This degeneracy was the source both of conceptual difficulties in defining a solution, and of difficulties in developing its asymptotics for small $\epsilon$. The concept of solution we used was that of a limit of solutions of nondegenerate problems. This was explained and studied in detail in Appendix B, where the most important properties of such solutions were also found. Two of these properties, (2.55) and (2.59), were crucial steps in developing the asymptotic reduction to a free-boundary problem.

Steady solutions of this free-boundary problem in the context of experiments with plates were investigated rigorously. We found a 'trailing' solution of Problem II (for a sufficiently thick plate) representing a grain boundary which lags behind the motion at the source (which is at the moving intersection of the grain boundary with the face of the plate), so that its shape becomes parallel to the face of the plate at a penetration distance of the order of $\frac{\delta}{\epsilon}$. Its speed is of the order $\epsilon^{2} D_{m} / \delta$.

We also found a 'connecting' solution of Problem I for plates with dimensionless thickness $H<\frac{1}{2} \sqrt{\rho}$, i.e. dimensional thickness $\hat{H}<\sqrt{\frac{D_{m} \tau}{4 \hat{\epsilon}\left(c^{\dagger}\right)^{2}}}$.

Finally, for $\rho \ll 1$ at a special plate thickness, there is a steady-moving grain boundary spanning the plate, which consists of a pair of trailing configurations as described, connected by a much wider bulge with width of the order $\frac{\delta}{\epsilon \rho}=\frac{\delta^{3}}{\hat{\epsilon}^{2}\left(c^{\dagger}\right)^{4} D_{m} \tau}$ (this is not rigorous). And if $\rho$ is small there are faster grain boundaries with speed at least as large as $\mathrm{O}\left(\frac{\epsilon^{3 / 2}}{\left(c^{\dagger}\right)^{3}} \sqrt{\frac{D_{m}}{\tau}}\right)$ spanning thin films of thickness $\mathrm{O}\left(\frac{\sqrt{\rho} \delta}{\epsilon}\right)$. As we shall see below, the bulging configuration may be so thick as to be unrealistic in practice.

The following reasonable but not universal rough orders of magnitudes for these quantities follow from the estimates given in [4]:

$$
\delta \approx 10^{-9} \mathrm{~m}, \quad D_{m} \tau \approx 10^{-19} \mathrm{~m}^{2}, \quad \hat{\epsilon} \approx 10^{-2}, \quad \hat{H} \approx 10^{-4} \mathrm{~m} .
$$

If we take the maximal concentration $c^{\dagger}$ to be $\approx 10^{-1}$, then

$$
\epsilon \approx 10^{-4}, \quad A \approx 10, \quad \rho \approx 10^{-5} .
$$


We may then check the validity of our basic assumptions (4.1) under the given estimates:

$$
\hat{\epsilon} \approx 10^{-2}, \quad c^{\dagger} \sqrt{\hat{\epsilon} A} \approx 10^{-3 / 2}, \quad \frac{\hat{\epsilon}\left(c^{\dagger}\right)^{2}}{A} \approx 10^{-5} .
$$

All of these numbers are $\ll 1$ as assumed, although the second one is perhaps marginal.

A typical penetration distance will then be $\frac{\delta}{\epsilon} \approx 10^{-5} \mathrm{~m}$, which is in agreement with experiments. The thickness of plates supporting the slow boundaries indicated in Section 3.4 may be of the order $\delta / \epsilon \rho \approx 1 \mathrm{~m}$. Under these parameter estimates, these travelling boundaries would be much too wide to be realizable in practice. However, this width is very sensitive to variations in $c^{\dagger}$, since it depends on the -4 power of that parameter. The fast configurations discussed in that section have dimensionless speed given by $\omega \approx \rho^{-1 / 2}$, or dimensional speed which could change a great deal depending on the temperature, a typical value being $\mathrm{O}\left(10^{-5}\right) \mathrm{m} \mathrm{s}^{-1}$.

When $\rho \ll 1$, one is tempted to approximate the free-boundary evolution problem (2.74), (2.75) by formally setting $\rho=0$. However, such a resulting problem is likely to be ill posed as an evolution problem. The small parameter $\rho$ apparently serves as a regularizing parameter for the ill-posed problem which must be kept, even though small.

Passing to the steady problem considered in Section 3, one has additional difficulties in setting $\rho=0$. In fact, in that case there will never be a (connecting) solution of Problem I, in which the grain boundary spans the plate, because (3.2) implies that every solution $\theta(s)$ is monotone. The grain boundary could not turn back to meet the other face. However, there is a reasonable solution of the infinite-plate problem (Theorem 1(a) allows that possibility); this is apparently the only case in which the approximation $\rho=0$ is reasonable when $\rho \ll 1$.

Many other problems suggest themselves by the treatment given here. The modelling can be extended to the motion of other grain boundary surfaces in a 3D material. Then the 'curvature' $\kappa$ in (2.74) will denote twice the mean curvature, and the diffusion term in (2.75) will be 2D diffusion in the surface. Corresponding plate problems with reservoir boundary conditions are formulated in a straightforward manner. For example, one analogue of the steady problem considered here would be the nonsteady expansion of a grain boundary which is radially symmetric with respect to an axis perpendicular to the plate.

The unidirectional nature of the model here, i.e. our assumption that $v>0(2.31)$, excludes many interesting phenomena such as double-seam configurations $[2,12]$. When a boundary begins to propagate into a grain which has already been changed by a DIGM motion, the basic equations will change somewhat, because the concentration $c_{0}$ appearing in (2.5) will have changed. Modelling and analysis can no doubt be performed to handle this situation as well.

The steady problem considered in Section 3 involved a source of solute at one or two boundaries of the moving grain boundary $\Gamma(t)$. One can also formulate problems in which $\Gamma$ is a closed curve and therefore has no boundaries or sources. For example, suppose it is a circle with changing radius, and that $u$ is uniform on the circle. The compatibility condition for initial conditions for (2.74), (2.75) then state that initially either $v=0$ or $u=0$. A transient solution such as in Section 2.7 will quickly bring arbitrary initial distributions to that condition. Whether $v$ or $u$ vanishes depends on whether the circle is expanding or contracting. In the former case, the transient brings it to rest with no further motion. In the latter, the circle will shrink to a point according to motion by curvature.

The asymptotic derivation of the free-boundary problem in this paper has recently been extended in [11] to some 3D situations, and applied to obtain a bidirectional model for the motion of grain 
boundaries spanning a thin metallic film. Theory and numerical simulations reflect the known phenomenon of double-seam formation and other phenomena.

\section{Acknowledgements}

PCFs research was supported by NSF Grant DMS-9703483. We are grateful to Oliver Penrose, who generously gave suggestions and ideas at every stage. We thank Geoffrey McFadden and Jean Taylor for critical reviews and valuable suggestions.

\section{REFERENCES}

1. Allen, S. M. \& CAHN, J. W. A microscopic theory for antiphase boundary motion and its application to antiphase domain coarsening. Acta Mater. 27, (1979) 1084-1095.

2. Baumann, S. F., Michael, J., \& Williams, D. B. Initiation and growth of the grain boundary discontinuous precipitation reaction. Acta Metall. 29, (1981) 1343-1355.

3. Cahn, J. W. \& Penrose, O. Theory of curvature and grooving effects in DIGM, in preparation

4. Cahn, J. W., Fife, P., \& Penrose, O. A phase field model for diffusion-induced grain boundary motion. Acta Mater. 45, (1997) 4397-4413.

5. Chen, X. \& Namah, G. Wave propagation under curvature effects in a heterogeneous medium. Applicable Anal. 64, (1997) 219-233.

6. Deckelnick, K., Elliott, C. M., \& Richardson, G. Long time asymptotics for forced curvature flow with applications to the motion of a superconducting vortex. Nonlinearity 10, (1997) 655-678.

7. Deckelnick, K. P., Elliott, C. M., \& Styles, V. M. Numerical diffusion induced grain boundary motion. Interfaces and Free Boundaries, to appear.

8. Deckelnick, K. P. \& Elliott, C. M. An existence and uniqueness result for phase field model of diffusion induced grain boundary motion. Proc. R. Soc. Edinburgh A, to appear.

9. Elliott, C. M. Approximation of curvature dependent interface motion. In: DUFF, I. S. \& WATson, G. A. (eds), State of the Art of Numerical Analysis. Clarendon Press, Oxford (1997) pp. 407-440.

10. Elliott, C. M., Gardiner, A. R., \& Kuhn, T. Generalized double obstacle phase field approximation of the anisotropic mean curvature flow, CMAIA Research Report 96-17, University of Sussex, (1996).

11. FIFE, P. C. \& WANG, X.-P. Chemically induced grain boundary dynamics, forced motion by curvature, and the appearance of double seams. Eur. J. Appl. Math., to appear.

12. Frebel, M. \& SChEnK, J. On the growth of the discontinuous precipitation reaction at individual grain boundaries in $\mathrm{Au}-40$ at.\% Fe alloys. Z. Metallkde. 70, (1979) 230-240.

13. HANDWERKER, C. A. Diffusion-induced grain boundary migration in thin films. In: GuPTA, D. \& Ho, P. S. (eds), Diffusion Phenomena in Thin Films and Microelectronic Materials. Noyes, Park Ridge, NJ (1988) pp. 245-322.

14. Hillert, M. \& Purdy, G. R. Chemically induced grain boundary motion. Acta Metall. 26, (1978).

15. JUdEN, J. N. Numerical solution of forced curvature flow for curves and a model for diffusion induced grain boundary motion, DPhil. University of Sussex, (1998).

16. MAYer, U. F. \& Simonett, G. Classical solutions for diffusion-induced grain-boundary motion. $J$. Math. Anal. Appl. 234, (1999) 660-674.

17. Mayer, U. F. \& Simonett, G. On diffusion-induced grain-boundary motion, Nonlinear Partial Differential Equations (Evanston, IL, 1998), Contemporary Mathematics 238, 231-240, Amer. Math. Soc., Providence, RI, 1999.

18. RHEE, W.-H. \& Yoon, D-N. The grain boundary migration induced by diffusional coherency strain in Mo-Ni alloys. Acta Metall. 37, (1989) 221.

19. Yoon, D. N. Chemically induced interface migration. Mater. Sci. Forum 193, (1996) 204-206. 


\section{Appendix A. Comparison of notation and assumptions}

The model considered in [3] is the same as that in our Section 3, namely (3.2) and (3.3), except that an additional effect of grooving is incorporated. form:

For reference, our equations (2.74), (2.75) take the following form when written in dimensional

$$
\begin{gathered}
\frac{\tau}{\delta} \hat{v}=\delta \hat{\kappa}+\hat{\epsilon} \hat{c}^{2}, \\
\hat{c}_{\hat{s} \hat{s}}-\frac{1}{\delta D_{m}} \hat{v} \hat{c}=0,
\end{gathered}
$$

where $\hat{c}$ is the value of the dimensional concentration midway through the grain boundary. They are equivalent to equations (1), (2) in [3] if we account for differences in the meaning of the symbols $u, c, s, \epsilon$ and set $\pi \bar{D}=D_{m}$. Our parameter $\hat{\epsilon}$ is the same as the parameter $\epsilon / \pi$ in [3].

More importantly, the nondimensionalizations in the two papers differ. Our dimensionless equations (3.2), (3.3) become (25), (24) in [3] if the following correspondances between the symbols in the two papers are noted:

\begin{tabular}{ll}
{$[3]$} & This paper \\
\hline$U$ & $u$ \\
$\theta$ & $\theta$ \\
$\cos \theta \frac{\mathrm{d}}{\mathrm{d} X}$ & $\rho \frac{\mathrm{d}}{\mathrm{d} s}$ \\
$q^{2}$ & $\rho$ \\
$C$ & $\rho^{2} \omega$ \\
$H_{0}$ & $H / \rho$ \\
$c_{b}-c_{0}$ & $c^{\dagger}$
\end{tabular}

Conjectures are made in the two papers about the maximal thicknesses of specimens admitting a steady-moving grain boundary spanning the faces. This is $H_{c}$ in our notation; see Section 3.3. It is proposed as a function of $\rho$. In the present paper, the conjecture is in order of magnitude only, but it agrees with that in [3]. On the other hand, a lower bound is proved in our Theorem 1(b). If $\rho$ is small, this lower bound is far from the conjectured value.

\section{Appendix B. Basic estimates and degenerate limits}

THEOREM 3 Part A. Let $\mathrm{d}(z)$ and $f_{i}(z), i=1, \ldots, 4$, be continuously differentiable on $(0,1)$ with $d(z)>0$ for $z \in(0,1)$, nondecreasing in $z$ for small $z$, and nonincreasing for $z$ near 1 . For some constant $m$, assume $\max _{z}\left|f_{i}(z)\right|+\max _{z}\left|f_{1}^{\prime}(z)\right| \leqslant m$ for each $i$. Let $\sigma_{i} \geqslant 0, i=2, \ldots, 5$ and $\sigma_{1}>0$. Let $W(z)$ be a function twice continuously differentiable on $(0,1)$ and also continuous at $z=0$. Let $\lim _{z \downarrow 0} d(z) W^{\prime}(z)=J<\infty$. Assume

$$
\begin{gathered}
\left(d(z) W^{\prime}(z)\right)^{\prime}+\sigma_{1}\left(\left(1+\sigma_{5} f_{1}(z)\right) W(z)\right)^{\prime}+\sigma_{2} f_{2}(z) d(z) W^{\prime}(z) \\
=\sigma_{3} d(z) f_{3}(z)+\sigma_{4} f_{4}(z), \quad z \in(0,1) .
\end{gathered}
$$

Assume that $\sigma_{2}, \sigma_{4}, \sigma_{5}$ are small enough, depending on $m$ and $M$, where $M=\max _{z}|W(z)|$.

There exists a constant $C>0$ depending only on $M$ and $m$ (and not on $d(z)$ ), such that for $0<z \leqslant \frac{1}{2}$,

$$
\left|W^{\prime}(z)\right| \leqslant C\left(\sigma_{2}+\sigma_{3}+\sigma_{5}+\frac{\sigma_{4}}{\sigma_{1}}+\frac{\sigma_{2}}{\sigma_{1}}|J|\right) .
$$


Part B. Now assume that the functions $d=d(z, s), f_{i}=f_{i}(z, s), W=W(z, s)$ depend also on another variable $s$ for small $s$, and are twice continuously differentiable in $(z, s)$. For each fixed $s$, we suppose the assumptions of part A are fulfilled uniformly for small $s$, and $J=0$. Also assume that for some continuously differentiable functions $h(z, s), h_{1}(z, s)$

$$
\partial_{s} d(z, s)=h(z, s) d(z, s), \quad \partial_{s}^{2} d(z, s)=h_{1}(z, s) d(z, s) .
$$

Then for all $z$

$$
d(z, s)\left|\partial_{s}^{k} W(z, s)-\partial_{s}^{k} W\left(\frac{1}{2}, s\right)\right| \leqslant C\left(\sigma_{1}+\sigma_{3}+\sigma_{4}\right), \quad k=0,1,2 .
$$

Proof. In the following, the symbols $f_{i j}$ will denote continuous functions of $z$ with $\max _{z}\left|f_{i j}(z)\right|$ bounded by a constant which depends only on $m$ and $M$, not on the parameters $\sigma_{i}$ and not on the function $d(z)$. For example, $f_{21}(z)=\exp \left[\sigma_{2} \int_{0}^{z} f_{2}\left(z^{\prime}\right) \mathrm{d} z^{\prime}\right]$ and $\left(f_{21}(z)\right)^{-1}$ are such functions, since $\sigma_{2}$ is small enough.

We multiply (B.1) by $f_{21}$ to obtain

$$
\left(f_{21}(z) d(z) W^{\prime}(z)\right)^{\prime}+\sigma_{1}\left(\left(1+\sigma_{5} f_{1}(z)\right) W\right)^{\prime} f_{21}(z)=\sigma_{3} d(z) f_{31}(z)+\sigma_{4} f_{41}(z)
$$

for functions $f_{31}=f_{3} f_{21}$ and $f_{41}=f_{4} f_{21}$.

Consider first the range $z<\frac{1}{2}$. It is seen that we may write

$$
\int_{0}^{z} d\left(z^{\prime}\right) f_{31}\left(z^{\prime}\right) \mathrm{d} z^{\prime}=d(z) f_{32}(z)
$$

because of the increasing nature of $d$ for small $z$. (Although $f_{32}$ depends on $d(z)$, its uniform bound does not.) Also, through integration by parts and the expression $f_{21}^{\prime}=\sigma_{2} f_{2} f_{21}$, we have $\int_{0}^{z}\left(\left(1+\sigma_{5} f_{1}(\zeta)\right) W\right)^{\prime} f_{21}(\zeta) \mathrm{d} \zeta=\left(1+\sigma_{5} f_{1}\right) f_{21}(z) W(z)-\left(1+\sigma_{5} f_{1}(0)\right) W(0)+\sigma_{2} f_{22}(z)$, where $f_{22}=-\int_{0}^{z} W f_{21} f_{2}\left(1+\sigma_{5}\right) f_{1} \mathrm{~d} \zeta$.

We now integrate (B.5) from 0 to $z$ to obtain

$$
\begin{gathered}
f_{21} d(z) W^{\prime}+\sigma_{1}\left[\left(1+\sigma_{5} f_{1}\right) f_{21} W-\left(1+\sigma_{5} f_{1}(0)\right) W(0)+\sigma_{2} f_{22}\right] \\
=J+\sigma_{3} d(z) f_{32}+\sigma_{4} \int_{0}^{z} f_{41} \mathrm{~d} \zeta .
\end{gathered}
$$

Divide by $f_{21}(z)$ to obtain

$$
\begin{gathered}
d(z) W^{\prime}+\sigma_{1}\left[\left(1+\sigma_{5} f_{1}\right) W-\left(1+\sigma_{5} f_{1}(0)\right) f_{21}^{-1} W(0)+\sigma_{2} f_{22} f_{21}^{-1}\right] \\
=J f_{21}^{-1}+\sigma_{3} d(z) f_{32} f_{21}^{-1}+\sigma_{4}\left(\int_{0}^{z} f_{41}\right) f_{21}^{-1} ; \\
d(z) W^{\prime}+\sigma_{1}\left[W-\left(1+\sigma_{5} f_{1}(0)\right) f_{21}^{-1} W(0)\right] \\
=J f_{21}^{-1}-\sigma_{1} \sigma_{5} f_{1} W-\sigma_{1} \sigma_{2} f_{22} f_{21}^{-1}+\sigma_{3} d(z) f_{32} f_{21}^{-1}+\sigma_{4}\left(\int_{0}^{z} f_{41}\right) f_{21}^{-1} \\
=J f_{21}^{-1}+\sigma_{1} \sigma_{5} f_{51}+\sigma_{1} \sigma_{2} f_{23}+\sigma_{3} d(z) f_{33}+\sigma_{4} f_{42} .
\end{gathered}
$$

Here $f_{51}=-f_{1} W, f_{23}=-f_{22} f_{21}^{-1}, f_{33}=f_{32} f_{21}^{-1}, f_{42}=\left(\int_{0}^{z} f_{41}\right) f_{21}^{-1}$. 
Now let

$$
Z(z)=W(z)-W(0)\left(1+\sigma_{5} f_{1}(0)\right) f_{21}^{-1}(z)-\sigma_{2} f_{23}(z)-\frac{\sigma_{4}}{\sigma_{1}} f_{42}(z)-\sigma_{5} f_{51}(z)-\frac{1}{\sigma_{1}} J f_{21}(z),
$$

so that by using $\partial_{z}\left(\left(f_{21}\right)^{-1}\right)=-\sigma_{2} f_{2} f_{21}^{-1}$ we have

$$
Z^{\prime}(z)=W^{\prime}(z)-\sigma_{2} f_{24}(z)-\frac{\sigma_{4}}{\sigma_{1}} f_{43}(z)-\sigma_{5} f_{52}(z)-\frac{\sigma_{2}}{\sigma_{1}} J f_{2} f_{21}^{-1}(z)
$$

where $f_{24}=\left[f_{2} f_{21}^{-1}\left(1+\sigma_{5} f_{1}(0)\right)+f_{23}^{\prime}\right], f_{43}=f_{42}^{\prime}, f_{52}=f_{51}^{\prime}$. Thus (B.7) becomes

$$
\left.d(z)\left[Z^{\prime}+\sigma_{2} f_{24}(z)+\frac{\sigma_{4}}{\sigma_{1}} f_{43}(z)+\sigma_{5} f_{52}(z)+\frac{\sigma_{2}}{\sigma_{1}} J f_{2} f_{21}^{-1}(z)\right)\right]+\sigma_{1} Z=\sigma_{3} d(z) f_{33}(z) .
$$

Divide by $d$ to obtain

$$
Z^{\prime}+\frac{\sigma_{1}}{d} Z=F
$$

where

$$
F=\sigma_{3} f_{33}-\sigma_{2} f_{24}-\frac{\sigma_{4}}{\sigma_{1}} f_{43}-\sigma_{5} f_{52}-\frac{\sigma_{2}}{\sigma_{1}} J f_{2} f_{21}^{-1}
$$

Multiply this equation by $\exp \left[\sigma_{1} \int_{1 / 2}^{z} \frac{d z^{\prime}}{d\left(z^{\prime}\right)}\right]$ and integrate, noting that $f_{42}(0)=f_{23}(0)=0$, $Z(0)=-\frac{J}{\sigma_{1}}$, to obtain

$$
Z(z)=-\frac{J}{\sigma_{1}}+\int_{0}^{z} F\left(z^{\prime}\right) \exp \left[-\sigma_{1} \int_{z^{\prime}}^{z} \frac{1}{d\left(z^{\prime \prime}\right)} \mathrm{d} z^{\prime \prime}\right] \mathrm{d} z^{\prime}
$$

hence

$$
Z^{\prime}(z)=F(z)-\frac{\sigma_{1}}{d(z)} \int_{0}^{z} F\left(z^{\prime}\right) \exp \left[-\sigma_{1} \int_{z^{\prime}}^{z} \frac{1}{d\left(z^{\prime \prime}\right)} \mathrm{d} z^{\prime \prime}\right] \mathrm{d} z^{\prime}
$$

Therefore

$$
\left|Z^{\prime}(z)\right| \leqslant C(m, M) \sup _{z}|F(z)|\left(1+\frac{\sigma_{1}}{d(z)} \int_{0}^{z} \exp \left[-\sigma_{1} \int_{z^{\prime}}^{z} \frac{1}{d\left(z^{\prime \prime}\right)} \mathrm{d} z^{\prime \prime}\right] \mathrm{d} z^{\prime}\right) .
$$

But for small $z, d(z)$ is nondecreasing, so that

$$
\frac{\sigma_{1}}{d(z)} \int_{0}^{z} \exp \left[-\sigma_{1} \int_{z^{\prime}}^{z} \frac{1}{d\left(z^{\prime \prime}\right)} \mathrm{d} z^{\prime \prime}\right] \mathrm{d} z^{\prime} \leqslant \frac{\sigma_{1}}{d(z)} \int_{0}^{z} \exp \left[-\frac{\sigma_{1}}{d(z)}\left(z-z^{\prime}\right)\right] \mathrm{d} z^{\prime}=1-\mathrm{e}^{-\frac{\sigma_{1} z}{d(z)}}<1
$$

so that

$$
\left|Z^{\prime}(z)\right| \leqslant C \sup _{z}|F(z)| .
$$


By (B.9) $W^{\prime}=Z^{\prime}-F+\sigma_{3} f_{33}$ so that

$$
\left|W^{\prime}(z)\right| \leqslant C\left(\sup _{z}|F(z)|+\sigma_{3}\right) .
$$

Again, although $Z$ and $W$ are affected by the function $d(z)$, the bounds on the right of (B.17) and (B.18) do not depend on $d$. The functions $f_{1}, f_{21}^{-1}, f_{23}, f_{43}, f_{51}, f_{33}$ and $f_{24}$, by their definitions are bounded in absolute value by $C(m, M)$. Similarly, $\left|f_{52}\right|=\left|-f_{1} W^{\prime}-f_{1}^{\prime} W\right| \leqslant C(m, M)\left(1+M^{\prime}\right)$, where

$$
M^{\prime}=\sup _{z \in(0,1 / 2]}\left|W^{\prime}(z)\right| .
$$

Thus $\sup _{z}|F(z)| \leqslant C\left(\sigma_{2}+\sigma_{3}+\sigma_{5}\left(1+M^{\prime}\right)+\frac{\sigma_{4}}{\sigma_{1}}+\frac{\sigma_{2}}{\sigma_{1}}|J|\right)$ and $M^{\prime} \leqslant C(m, M)\left(\sigma_{2}+\sigma_{3}+\sigma_{5}(1+\right.$ $\left.\left.M^{\prime}\right)+\frac{\sigma_{4}}{\sigma_{1}}+\frac{\sigma_{2}}{\sigma_{1}}|J|\right)$. Since $\sigma_{5} \ll 1$, we obtain

$$
M^{\prime} \leqslant C(m, M)\left(\sigma_{2}+\sigma_{3}+\sigma_{5}+\frac{\sigma_{4}}{\sigma_{1}}+\frac{\sigma_{2}}{\sigma_{1}}|J|\right),
$$

which yields (B.2).

Finally, consider part B. We now write (B.7) in the form

$$
d(z, s) \partial_{z} W(z, s)=\sigma_{1} f_{11}(z, s)+\sigma_{3} d(z, s) f_{33}(z, s)+\sigma_{4} f_{42}(z, s),
$$

where $f_{11}=\sigma_{5} f_{51}+\sigma_{2} f_{23}-\left[W-\left(1+\sigma_{5} f_{1}(0)\right) f_{21}^{-1} W(0)\right]$. The functions $f_{i j}$ are defined as before, but depend now on $s$ as well.

Differentiate (B.20) with respect to $s$ to obtain

$$
\partial_{s}\left(d(z, s) \partial_{z} W\right)=\partial_{s}\left[\sigma_{1} f_{11}+\sigma_{3} d(z, s) f_{33}+\sigma_{4} f_{42}\right] .
$$

Hence, using (B.3),

$$
d(z, s) \partial_{z} \partial_{s} W=\partial_{s}\left[\sigma_{1} f_{11}+\sigma_{3} d(z, s) f_{34}+\sigma_{4} f_{44}\right]-h(z, s) d(z, s) \partial_{z} W .
$$

We substitute for the quantity $d(z, s) \partial_{z} W$ appearing in the last term of (B.21) the right side of (B.20) to obtain the estimate

$$
d(z, s)\left|\partial_{s} \partial_{z} W\right|<C\left(\sigma_{1}+\sigma_{3}+\sigma_{4}\right) .
$$

We differentiate (B.21) a second time with respect to $s$ and use similar calculations to obtain

$$
d(z, s)\left|\partial_{s}^{2} \partial_{z} W\right|<C\left(\sigma_{1}+\sigma_{3}+\sigma_{4}\right) .
$$

It suffices to prove (B.4) for $z<\frac{1}{2}$ in the interval where $d(z)$ is nondecreasing, or the analogous interval near $z=1$, for otherwise $d$ is bounded from below, so we may divide (B.22), (B.23) by $d(z, s)$ and integrate. So let $z$ be near 0 . We have, by dividing (B.22) by $z$, integrating from $z=\frac{1}{2}$, and using the monotonicity of $d$,

$$
\left|\partial_{s} W(z, s)-\partial_{s} W\left(\frac{1}{2}, s\right)\right| \leqslant C\left(\sigma_{1}+\sigma_{3}+\sigma_{4}\right) \int_{z}^{1 / 2} \frac{\mathrm{d} \zeta}{\mathrm{d}(\zeta)} \leqslant C\left(\sigma_{1}+\sigma_{3}+\sigma_{4}\right) \frac{1}{d(z)}\left(\frac{1}{2}-z\right),
$$

and (B.4) follows for $k=1$. The proofs for $k=0$ and 2 are the same. 
THEOREM 4 Let $d_{n}(z)$, for $n=1,2, \ldots$ be a sequence of smooth functions defined for $-a<z<$ 1 , for some $a>0$. They satisfy $d_{n}(z)>\delta_{n}>0$, are nondecreasing for $-a<z<a$, nonincreasing for $1-a<z<1$, and $\lim _{n \rightarrow \infty} d_{n}(z)=d^{*}(z)$, where $d^{*}(z)=0$ for $-a<z<0, d^{*}(z)>0$ for $0<z<1$. We assume the convergence is uniform, together with their first derivatives, in any interval $\left[\alpha_{1}, \alpha_{2}\right] \subset(0,1)$, so that $d^{*}$ satisfies the hypotheses of Theorem 3 .

Let the functions $f_{i}$ and constants $\sigma_{i}, m$ satisfy the hypotheses of Theorem 3, except that the $f_{i}$ are defined for $-a<z<1$. The functions $f_{i}=f_{i}^{(n)}$ may depend on $n$, but they are to be equicontinuous in $n$.

Let $W_{n}(z)$ be a sequence of functions defined on $(-a, 1)$ satisfying (B.1) with $d$ replaced by $d_{n}$, $\sup _{z}\left|W_{n}(z)\right|<M$ for all $n$, and $J_{n}=\lim _{z \downarrow-a} d_{n}(z) W_{n}^{\prime}(z)$ bounded independently of $n$.

Then there exists a subsequence of the $W_{n}$ which converges to a limit function $W(z)$ uniformly in each interval $\left(-a, z_{2}\right)$ for any $z_{2}<1$, and $\lim _{n \rightarrow \infty} J_{n}=0$. The limit function $W$ is twice continuously differentiable on the half-closed interval $[0,1)$ and satisfies (B.1) with $d=d^{*}$ and with functions $f_{i}$ which are limits of the $f_{i}^{(n)}$, (B.2) with $J=0$, and (B.4).

Note. The limit $d^{*}$ may be discontinuous at $z=0$.

Proof. Let $Z_{n}$ be defined by (B.8) with subscripts $n$. The functions $f_{i j}^{(n)}$ are bounded and equicontinuous in $n$. The sequence of functions on the right of (B.13) is equicontinuous in $n$ for each interval $\left(-a, z_{2}\right)$. This would be obvious except for (a) the fact that by construction some of the functions $f_{i j}^{(n)}$ depend on the undifferentiated functions $W_{n}$, hence on $Z_{n}$, and (b) the possible singularity of the exponential factor

$$
\exp \left[-\sigma_{1} \int_{z^{\prime}}^{z} \frac{1}{d_{n}\left(z^{\prime \prime}\right)} \mathrm{d} z^{\prime \prime}\right]
$$

in the integrand of (B.13). This turns out not to destroy equicontinuity. To show this, it suffices to show that the sequence of functions

$$
G_{n}(z)=\int_{0}^{z} \exp \left[-\sigma_{1} \int_{z^{\prime}}^{z} \frac{1}{d_{n}\left(z^{\prime \prime}\right)} \mathrm{d} z^{\prime \prime}\right] \mathrm{d} z^{\prime}
$$

is equicontinuous for $z$-intervals bounded away from $z=1$. Differentiating, we find

$$
G_{n}^{\prime}(z)=1-\frac{\sigma_{1}}{d_{n}(z)} G_{n}(z)<1 .
$$

But for small $z$ by the monotonicity of $d_{n}$ we have $G_{n}(z)<\int_{0}^{z} \exp \left[-\frac{\sigma_{1}}{d_{n}(z)} \int_{z^{\prime}}^{z} \mathrm{~d} z^{\prime \prime}\right] \mathrm{d} z^{\prime}=$ $\int_{0}^{z} \exp \left[-\frac{\sigma_{1}}{d_{n}(z)} \tau\right] \mathrm{d} \tau<\frac{d_{n}(z)}{\sigma_{1}}$. We put this into (B.25) to obtain

$$
0<G_{n}^{\prime}(z)<1,
$$

from which the equicontinuity for small $z$ follows. For intermediate values of $z$, it is straightforward. The function is not generally equicontinuous on intervals which include $z=1$.

Regarding (a), these functions are multiplied in (B.13) by small parameters; after being so multiplied, the parts of their moduli of continuity due to the variation of $Z$ are small compared with the modulus of continuity of the left side of that equation, namely $Z$, and can be disregarded. 
In view of the equicontinuity, there exists a subsequence $Z_{n} \rightarrow Z$, the convergence being uniform, and $J_{n} \rightarrow J$. Moreover, by the hypotheses on the $d_{n}$, for $z \in(0,1)$ the right side of (B.13) (with subscripts) converges to the same expression with $d=d^{*}$, uniformly for $z \in\left[\alpha_{1}, \alpha_{2}\right] \subset(0,1)$. We define $W$ in terms of $Z$ by (B.8), so by (B.8), $W$ satisfies (B.7) for each $z \in(0,1)$. Reversing the steps from (B.5) to (B.7), we verify that (B.5) is satisfied with $d=d^{*}$ for $z \in(0,1)$. By (B.14), the functions $W_{n}^{\prime}$ also converge uniformly to the limit $W^{\prime}$ on $(-a, 1-\delta)$. Therefore, for any $z \in(-a, 0)$, $0=d^{*}(z) W^{\prime}(z)=\lim _{n \rightarrow \infty} d_{n}(z) W^{\prime}(z)$. Since $d^{*}(z) W^{\prime}(z)$ is continuous, it follows that the number $J=0$ in Theorem 3 part A.

In short, the function $W$ satisfies the hypotheses of Theorem 3 with $J=0$, and the resulting estimates hold.

Commentary on the significance of Theorem 4. The main point of mathematical difficulty, by far, in our asymptotic reduction to a free-boundary problem was the fact that the diffusivity $D(\phi)$ is zero outside the grain boundary. A similar problem would arise if it were positive but very small. The partial differential equation (2.11) (or in another form, (2.51)) therefore becomes degenerate at the trailing edge $r=z=0$ and also at the leading edge. The properties, and even the existence, of solutions near the degeneracy may be subject to question.

With the aid of Theorem 4 we conceive of solutions $W$ of our degenerate PDE (2.51), recast in the form (B.1), as being limits of solutions of nondegenerate ones. This is assumption (2.29). We may take the approximating sequence $d_{n}(z)$ of diffusivities to be strictly positive and continuous everywhere, as long as each satisfies the monotonicity requirements of Theorem 3 .

If we assume that for each $n$ there is a solution of the nondegenerate problem which is bounded independently of $n$ and equicontinuous in $(s, t)$, then the theorem ensures the existence of a limit solution of the degenerate problem which is continuous even at the point $z=0$ of degeneracy. This argument, by the way, applies only at the trailing edge, not at the leading edge; we rely on $v$ in (2.51) being positive, and so obtain a unidirectional result.

In our modelling process, we understand the concentration function $w$ or $W$ of the degenerate problem (2.11) or (2.51) to be such a limiting solution. Its continuity at the trailing edge $\Gamma$ is then guaranteed.

Given that, the estimates provided by Theorem 3 then lead to (2.55) and (2.56), which show that the solution has uniform behaviour near the trailing edge, and also supply the central estimates needed to effect a reduction to our free-boundary problem.

\section{Appendix C. Details in the derivation to $\mathrm{O}(\epsilon)$}

The purpose of this appendix is to derive (2.79), (2.78) when $D=\frac{1}{\pi}=d(z), \rho=1$, and $c^{\dagger}=1$, so that $\hat{\epsilon}$ and $\epsilon=\pi \hat{\epsilon}$ have a common order of magnitude. First, some improved estimates on the variation of $U$.

In the present case $D=$ const, and a sharper estimate than (2.55) can be obtained. In fact (2.51) and $J=0$ become

$$
\partial_{z}^{2} W=\epsilon \kappa \partial_{z} W+\mathrm{O}\left(\epsilon^{2}\right), \quad \partial_{z} W(0)=0 .
$$

And this relation may be differentiated with respect to $s$. An expansion $W=W_{0}+\epsilon W_{1}+\cdots$ reveals that $W_{0}(z, s, t)=W_{0}(s, t)$ is independent of $z$ and the same is true of $W_{1}$. Hence, in place 
of (2.55) we have

$$
\left|W(z, s, t)-W_{0}(s, t)\right| \leqslant C \epsilon^{2}
$$

on the entire range of $z<z_{1}$.

Let $g(z)=\frac{\pi}{4}(1-\cos z)^{2}, g_{1}=g(\pi / 2)=\frac{\pi}{4}$. From (2.26), (C.2), and (2.39),

$$
W=W_{0}+\mathrm{O}\left(\epsilon^{2}\right)=U(z)(1+\epsilon g(z))+\mathrm{O}\left(\epsilon^{2}\right) .
$$

We express $U=U_{0}+\epsilon U_{1}+\mathrm{O}\left(\epsilon^{2}\right)$ and insert this into (C.3) to get

$$
U_{0}=W_{0}, \quad U_{1}(z)=-U_{0} g(z), \quad U=U_{0}(1-\epsilon g(z))+\mathrm{O}\left(\epsilon^{2}\right),
$$

where $U_{0}$ and $W_{0}$ are independent of $z$. Thus, the function in (2.73) is

$$
u(s, t)=U(\pi / 2, s, t)=U_{0}(s, t)\left(1-\epsilon g_{1}\right)+\mathrm{O}\left(\epsilon^{2}\right)
$$

and

$$
W(z, s, t)=u(s, t)\left(1+\epsilon g_{1}\right)+\mathrm{O}\left(\epsilon^{2}\right),
$$

which may be differentiated with respect to $s$ (note $g_{1}$ is independent of $s$ ).

Differentiating (C.1) with respect to $s$, we get

$$
\partial_{s} W_{z z}=\epsilon \kappa \partial_{s} W_{z}+\epsilon \kappa_{s} W_{z}+\mathrm{O}\left(\epsilon^{2}\right) .
$$

So for the same reason that (C.2) is valid,

$$
\left|\partial_{s} W(z, s, t)-\partial_{s} W_{0}(s, t)\right|<C \epsilon^{2} .
$$

We now look into the derivation of (2.78). We do the expansions $\Phi(\zeta)=\Phi^{0}(\zeta)+\epsilon \Phi^{1}(\zeta)+$ $\epsilon^{2} \Phi(\zeta)+\cdots, v=v^{0}+\epsilon v^{1}+\cdots, \zeta=\left(1+\epsilon \alpha^{0}+\epsilon^{2} \alpha^{1} \ldots\right) z$ where $\Phi^{0}=-\cos \zeta$.

By (C.4), (C.5) the function $p_{\phi}$ appearing in (2.33), (2.43) takes the form

$$
\begin{gathered}
p_{\phi}=\frac{\pi}{4}(1+\Phi) U_{0}^{2}(1-2 \epsilon g(z))+\mathrm{O}\left(\epsilon^{2}\right)=\frac{\pi}{4}(1+\Phi) u^{2}\left(1+2 \epsilon g_{1}\right)(1-2 \epsilon g(z))+\mathrm{O}\left(\epsilon^{2}\right) \\
=\frac{\pi}{4}(1+\Phi) u^{2}\left(1-2 \epsilon\left(g_{1}-g(z)\right)\right)+\mathrm{O}\left(\epsilon^{2}\right) .
\end{gathered}
$$

and (2.44) becomes $\left(v=v^{*}, s=s^{*}\right)$

$$
v^{0}(x, t)=\kappa(s, t)+u^{2}(s, t),
$$

so that the higher-order expansion corresponding to (2.33) is

$$
\begin{aligned}
\Phi_{\zeta \zeta}+\Phi= & \epsilon\left(-v \Phi_{\zeta}+\kappa\left(1+\epsilon f_{1}\right) \Phi_{\zeta}\right)+\frac{\pi}{4} \epsilon(1+\Phi) u^{2}\left(1-2 \epsilon\left(g_{1}-g(\zeta)\right)\right. \\
& +\epsilon^{2} \Phi_{t}+\epsilon^{2}\left(1+\epsilon f_{2}\right) \partial_{s}\left[\left(1+\epsilon f_{2}\right) \Phi_{s}\right],
\end{aligned}
$$

for some regular functions $f_{1}(\zeta)$ and $f_{2}(\zeta)$. Substituting (C.10) into the $\mathrm{O}(1)$ term in (2.40), we find (analogous to (2.40))

$$
\Phi_{\zeta \zeta}^{1}+\Phi^{1}=u^{2}\left(-\sin \zeta+\frac{\pi}{4}(1-\cos \zeta)\right)-2 \alpha \cos \zeta,
$$


so that the particular solution seen in (2.45) takes the form $\Phi^{\dagger}=u^{2} H_{1}(\zeta)$ with $H_{1}$ independent of any parameters. It is then easily seen from the analysis subsequent to (2.45) that

$$
\alpha=u^{2} \hat{\alpha}, \quad \gamma=u^{2} \hat{\gamma}, \quad \Phi^{1}(\zeta)=u^{2} \hat{\Phi}^{1}(\zeta),
$$

again with the roofed quantities independent of any parameters and of $u$. Expanding (C.11) to next order with use of (C.12), we have

$$
\Phi_{\zeta \zeta}^{2}+\Phi^{2}=-v^{1} \sin \zeta+h_{2}(\zeta) u^{4}+h_{3}(\zeta) u^{2}+\kappa h_{4}(\zeta)-2 \alpha \cos \zeta
$$

where $h_{2}(\zeta)=-2 \hat{\alpha_{0}} \hat{\Phi}_{\zeta \zeta}^{1}-\hat{\Phi}_{\zeta}^{1}-\hat{\alpha}^{0} \sin \zeta+\hat{\Phi}^{1}$, etc. The right side of (C.13) must be orthogonal to $\cos \zeta$. Applying this condition results in

$$
v^{1}=A u^{4}+B u^{2}+C \kappa,
$$

for coefficients $A, B, C$ which can be calculated in principle. Thus except for an $\mathrm{O}\left(\epsilon^{2}\right)$ error,

$$
v=v^{0}+\epsilon v^{1}=\kappa(1+\epsilon C)+u^{2}\left(1+\epsilon\left(B+A u^{2}\right)\right),
$$

which yields (2.78).

Next, we derive (2.79), first developing an expression for $\frac{\mathrm{d}}{\mathrm{d} t} M(0)$. We shall obtain an expression similar to (D.10).

Let $\mathrm{d} t$ be a small time increment. We have, from (2.61) and (2.18), using the same definition (D.7) of $\bar{x}$, the following representation, analogous to (D.9):

$$
M(\mathrm{~d} t)=\epsilon \int_{s_{1}}^{s_{2}} \int_{0}^{\pi} U\left(z, s^{\prime}, \mathrm{d} t\right)\left|\frac{\mathrm{d} \bar{s}}{\mathrm{~d} s}\right| \mathrm{d} z \mathrm{~d} s^{\prime}(1+\mathrm{O}(\epsilon)) .
$$

Now from (2.57) with $d=$ const and (2.73),

$$
U(z, s, t)=u(s, t)+\mathrm{O}(\epsilon) .
$$

Therefore

$$
M(\mathrm{~d} t)=\epsilon \pi \int_{s_{1}}^{s_{2}} u(s, \mathrm{~d} t)(1+\mathrm{d} t v(s, 0) \kappa(s, 0)) \mathrm{d} s+\mathrm{O}\left(\epsilon^{2}\right) .
$$

Again taking the derivative, we obtain

$$
\frac{\mathrm{d}}{\mathrm{d} t} M(0)=\epsilon \pi \int_{s_{1}}^{s_{2}}\left(u_{t}+u v \kappa\right) \mathrm{d} s+\mathrm{O}\left(\epsilon^{2}\right) .
$$

We now consider $F_{1}(0)$ (2.64). From (C.5) and $g(0)=0$, we have

$$
F_{1}(0)=-\left(1+\epsilon g_{1}\right) \int_{s_{1}}^{s_{2}} v(s, 0) u(s, 0) \mathrm{d} s+\mathrm{O}\left(\epsilon^{2}\right) .
$$

Consider next $F_{2}(0)$. As in (2.67), use the fact (Section 2.3.1) that the thickness of $G$ is $\pi(1+$ $\left.\epsilon u^{2} \hat{\alpha}^{0}\right)+\mathrm{O}\left(\epsilon^{2}\right)$ from (C.12). Thus since $D=1 / \pi$,

$$
\begin{gathered}
F_{2}=\left.\frac{1}{\pi} \int_{0}^{\pi\left(1+\epsilon u^{2} \hat{\alpha}^{0}\right)} W_{s}(z, s, t) \mathrm{d} z\right|_{s=s_{2}} ^{s=s_{1}} \\
=\left.\frac{1}{\pi} \int_{0}^{\pi} W_{s}\left(\zeta\left(1+\epsilon u^{2} \hat{\alpha}^{0}\right), s, t\right)\left(1+\epsilon u^{2} \hat{\alpha}^{0}\right) \mathrm{d} \zeta\right|_{s=s_{2}} ^{s=s_{1}} .
\end{gathered}
$$


We use the $s$-differentiated version of (C.6) to obtain

$$
\begin{aligned}
F_{2} & =\left.u_{s}(s, t)\left(1+\epsilon g_{1}\right)\left(1+\epsilon u^{2} \hat{\alpha}^{0}\right)\right|_{s_{2}} ^{s_{1}}+\mathrm{O}\left(\epsilon^{2}\right) \\
& =\left(1+\epsilon g_{1}\right) \int_{s_{1}}^{s_{2}}\left(\left(1+\epsilon u^{2} \hat{\alpha}^{0}\right) u_{s}\right)_{s} \mathrm{~d} s .
\end{aligned}
$$

Using (2.69) again with (C.18)-(C.20) and the arbitrariness of $s_{1}$ and $s_{2}$, we get

$$
\epsilon \pi\left(u_{t}+u v \kappa\right)+u v\left(1+\epsilon g_{1}\right)=\left(1+\epsilon g_{1}\right)\left(u_{s}\left(1+\epsilon u^{2} \hat{\alpha}^{0}\right)\right)_{s}+\mathrm{O}\left(\epsilon^{2}\right),
$$

or (neglecting the error term)

$$
u_{t}=\frac{1}{\epsilon^{*}}\left(u_{s}\left(1+\epsilon u^{2} \hat{\alpha}^{0}\right)_{s}-\frac{1}{\epsilon^{*}} v u-u v \kappa,\right.
$$

where $\epsilon^{*}=\frac{\epsilon \pi}{1+g_{1} \epsilon}$. This yields (2.79).

\section{Appendix D. Details in the curvilinear coordinate transformation}

Recall that $\boldsymbol{\nu}(x)$ is the unit vector directed from $X(x)$ toward $x$, and $\tau(x)$ is the one obtained by rotating $\boldsymbol{\nu}(x)$ through the angle $\pi / 2$. Then the spatial gradients are

$$
\nabla r(x, 0)=\boldsymbol{\nu}(x), \quad \nabla s(x, 0)=\frac{1}{1-r(x) \kappa(x)} \tau(x),
$$

where $\kappa(x)$ is the curvature of $\Gamma_{-}(0)$ at the point $X(x)$. The reason for (D.1) is the following. Since $s$ is constant on rays perpendicular to $\Gamma_{-}(0)$, i.e. in the direction of $\boldsymbol{\nu}(x), \nabla s(x, 0)$ is in the direction of $\tau$. Its magnitude is, by dilation due to curvature, inversely proportional to distance from the centre of curvature, i.e. to $\kappa^{-1}(x)-r(x, 0)$, and the magnitude is unity on $\Gamma_{-}(0)$, i.e. for $r=0$. These conditions imply (D.1). In terms of the coordinates $(r, s)$, we have that $\boldsymbol{\nu}, \boldsymbol{\tau}, \kappa$ are functions $\boldsymbol{\nu}(s)$, $\tau(s), \kappa(s)$.

Therefore, using subscripts to denote partial derivatives with respect to the coordinates $r$ and $s$, we have

$$
\begin{gathered}
\boldsymbol{\nu} \cdot \boldsymbol{\tau}=0, \quad(\nabla r)_{r}=0, \quad(\nabla s)_{r}=\frac{\kappa(s)}{(1-r \kappa(s))^{2}} \tau, \quad(\nabla r)_{s}=-\kappa(s) \tau, \\
(\nabla s)_{s}=\frac{r \kappa_{s}(s)}{(1-r \kappa(s))^{2}} \tau+\frac{\kappa(s)}{1-r \kappa(s)} \nu .
\end{gathered}
$$

We also denote by $\partial_{\tau}$ and $\partial_{\nu}$ the directional derivatives in the directions of $\tau$ and $\nu$, and use the representation (since $r=\epsilon z, s=\rho^{1 / 2} s^{*}$ )

$$
\nabla w=\boldsymbol{\nu} \partial_{\nu} w+\boldsymbol{\tau} \partial_{\tau} w=\boldsymbol{\nu} \epsilon^{-1} \partial_{z} W+\frac{1}{1-\epsilon z \kappa} \boldsymbol{\tau} \rho^{-1 / 2} \partial_{s^{*}} W .
$$


Similarly,

$$
\begin{gathered}
\nabla \cdot D \nabla w=\boldsymbol{\nu} \cdot \partial_{\nu}\left[\boldsymbol{\nu} \epsilon^{-1} D \partial_{z} W+\frac{D}{1-\epsilon z \kappa} \tau \rho^{-1 / 2} \partial_{s^{*}} W\right] \\
+\boldsymbol{\tau} \cdot \frac{1}{1-\epsilon z \kappa} \rho^{-1 / 2} \partial_{s^{*}}\left[\boldsymbol{\nu} \epsilon^{-1} D \partial_{z} W+\frac{D}{1-\epsilon z \kappa} \tau \rho^{-1 / 2} \partial_{s^{*}} W\right] \\
=\boldsymbol{\nu} \cdot\left[\epsilon^{-2} \partial_{z} D W_{z} \boldsymbol{\nu}+\boldsymbol{\tau} \epsilon^{-1} \partial_{z}\left(\rho^{-1 / 2} \frac{D W_{s^{*}}}{1-\epsilon z \kappa}\right)+0\right] \\
+\frac{1}{1-\epsilon z \kappa} \boldsymbol{\tau} \cdot\left[\boldsymbol{\nu} \epsilon^{-1} \rho^{-1 / 2} \partial_{s^{*}}\left(D W_{z}\right)+D \epsilon^{-1} W_{z}(-\kappa \boldsymbol{\tau})+\rho^{-1 / 2} \frac{D W_{s^{*}}}{1-\epsilon z \kappa} \kappa \boldsymbol{\nu}+\rho^{-1} \partial_{s^{*}}\left(\frac{D W_{s^{*}}}{1-\epsilon z \kappa}\right) \boldsymbol{\tau}\right] \\
=\epsilon^{-2}\left(D W_{z}\right)_{z}-\frac{\epsilon^{-1} \kappa}{1-\epsilon z \kappa} D W_{z}+\frac{\rho^{-1}}{1-\epsilon z \kappa} \partial_{s^{*}}\left(\frac{D W_{s^{*}}}{1-\epsilon z \kappa}\right) .
\end{gathered}
$$

This represents the right side of (2.25) at $t=0$. The left side is derived in Section 2.2.

One last thing to do is to characterize $s_{t}(x, 0)$ in terms of more familiar things, namely (2.20). Consider a small time interval $\mathrm{d} t$. We consider the mapping from $\Gamma_{-}(0)$ to $\Gamma_{-}(\mathrm{d} t)$ by normal projection, namely the function

$$
\bar{x}(s)=x(0, s)+\nu(s) v(s, 0) \mathrm{d} t,
$$

which maps points on $\Gamma_{-}(0)$ (given by values of $s$ ) to points $\bar{x}(s)$ on $\Gamma_{-}(\mathrm{d} t)$. Now continuing to denote points on $\Gamma_{-}(\mathrm{d} t)$ by $\bar{x}$, we have

$$
s(x, \mathrm{~d} t)= \pm \int_{Y \cap \Gamma_{-}(\mathrm{d} t)}^{X(x, \mathrm{~d} t)}|\mathrm{d} \bar{x}|,
$$

where the integral is over the indicated portion of $\Gamma_{-}(\mathrm{d} t)$, oriented positively in the direction of $\tau$, and the + sign is chosen if $X(x, \mathrm{~d} t)$ is on the positive side of $Y \cap \Gamma_{-}(\mathrm{d} t)$. Changing variables and denoting the arclength integration variable on $\Gamma_{-}(0)$ by $s^{\prime}$, we transform this to

$$
s(x, \mathrm{~d} t)=\int_{X_{0}}^{X(x, 0)}\left|\frac{\mathrm{d} \bar{x}}{\mathrm{~d} s^{\prime}}\right| \mathrm{d} s^{\prime} .
$$

But $\left|\frac{\mathrm{d} \bar{x}}{\mathrm{~d} s}\right|$ is the dilatation due to curvature mentioned above, so that $\left|\frac{\mathrm{d} \bar{x}}{\mathrm{~d} s}\right|=1+\kappa(s) \mathrm{d} r(s)=$ $1+v(s) \kappa(s) \mathrm{d} t$. Therefore taking the time derivative, we find

$$
s_{t}(x, 0)=\int_{0}^{s} v\left(s^{\prime}, 0\right) \kappa\left(s^{\prime}, 0\right) \mathrm{d} s^{\prime} .
$$

We set $v(s, t)=\rho^{-1} v^{*}\left(s^{*}, t\right)$ to obtain (with $s^{\prime}=\rho^{1 / 2} \bar{s}$ )

$$
\begin{aligned}
s_{t}(x, 0)= & \rho^{-1} \int_{0}^{s} v^{*}\left(\rho^{-1 / 2} s^{\prime}, 0\right) \kappa\left(s^{\prime}, 0\right) \mathrm{d} s^{\prime} \\
& =\rho^{-1} S(s, 0),
\end{aligned}
$$


where

$$
S(s, 0)=\int_{0}^{s} v^{*}\left(\rho^{-1 / 2} s^{\prime}, 0\right) \kappa\left(s^{\prime}, 0\right) \mathrm{d} s^{\prime} .
$$

For other times $t$, this generalizes to (2.20)-(2.22).

If assumptions I and IV hold (Section 2.3), then we have the estimate

$$
|S(s, t)| \leqslant C \int_{0}^{s}\left|\kappa\left(s^{\prime}, 0\right)\right| \mathrm{d} s^{\prime} \leqslant C .
$$

